\title{
HEIGHT ZETA FUNCTIONS OF TORIC VARIETIES
}

\author{
VICTOR V. BATYREV AND YURI TSCHINKEL
}

\begin{abstract}
We investigate analytic properties of height zeta functions of toric varieties. Using the height zeta functions, we prove an asymptotic formula for the number of rational points of bounded height with respect to an arbitrary line bundle whose first Chern class is contained in the interior of the cone of effective divisors.
\end{abstract}

Supported by Deutsche Forschungsgemeinschaft. Leibniz Fellow at ENS, Paris. 
Contents

1. Introduction 2

2. Technical theorems 4

3. Fourier analysis on algebraic tori 13

4. Geometry of toric varieties 16

5. Analytic properties of height zeta functions 20

6. Appendix: $\mathcal{X}$-functions of polyhedral cones 26

$\begin{array}{ll}\text { References } & 27\end{array}$

\section{INTRODUCTION}

Let $X$ be a $d$-dimensional algebraic variety defined over a number field $F$. Denote by $\mathcal{L}=\left(L,\left\{\|\cdot\|_{v}\right\}\right)$ a metrized line bundle on $X$, i.e. a line bundle $L$ together with a family of $v$-adic metrics, where $v$ runs over the set $\operatorname{Val}(F)$ of all valuations of $F$. For any locally closed algebraic subset $Y \subset X$ we denote by $Y(F)$ the set of $F$-rational points in $Y$. A metrized line bundle $\mathcal{L}$ defines a height function

$$
H_{\mathcal{L}}: X(F) \rightarrow \mathbf{R}_{>0} .
$$

Assume that a subset $Y \subset X$ and a bundle $L$ are choosen in such a way that

$$
N(Y, \mathcal{L}, B):=\#\left\{x \in Y(F) \mid H_{\mathcal{L}}(x) \leq B\right\}<\infty
$$

for all $B \in \mathbf{R}_{>0}$ (e.g., this holds for any $Y \subset X$ if $L$ is ample). Then the asymptotic behavior of the counting function $N(Y, \mathcal{L}, B)$ as $B \rightarrow \infty$ is determined by analytic properties of the height zeta function $Z(Y, \mathcal{L}, s)$ defined by the series

$$
Z(Y, \mathcal{L}, s):=\sum_{x \in Y(F)} H_{\mathcal{L}}(x)^{-s}
$$

which converges for $\operatorname{Re}(s) \gg 0$. More precisely, one has the following Tauberian statement:

Theorem 1.1. [2] Assume that the series $Z(Y, \mathcal{L}, s)$ is absolutely convergent for $\operatorname{Re}(s)>a>0$ and that there exists some positive integer $b$ such that

$$
Z(Y, \mathcal{L}, s)=\frac{g(s)}{(s-a)^{b}}+h(s)
$$


where $g(s)$ and $h(s)$ are functions holomorphic in the domain $\operatorname{Re}(s) \geq a$ and $g(a) \neq 0$. Then the following asymptotic formula holds:

$$
N(Y, \mathcal{L}, B)=\frac{g(a)}{a(b-1) !} B^{a}(\log B)^{b-1}(1+o(1)) \text { for } B \rightarrow \infty .
$$

Definition 1.2. Denote by $N S(X)$ be the Neron-Severi group of $X$ and by $N S(X)_{\mathbf{R}}=N S(X) \otimes \mathbf{R}$. The cone of effective divisors of $X$ is the closed cone $\Lambda_{\text {eff }}(X) \subset N S(X)_{\mathbf{R}}$ generated by the classes of effective divisors.

Let $[L] \in N S(X)$ be the first Chern class of $L$. Denote by $\mathcal{K}_{X}=$ $\left(K_{X},\left\{\|\cdot\|_{v}\right\}\right)$ the metrized canonical line bundle on $X$.

Definition 1.3. Let $L$ be any line bundle on $X$. Define

$$
a(L):=\inf \left\{a \in \mathbf{R} \mid a[L]+\left[K_{X}\right] \in \Lambda_{\text {eff }}(X)\right\} .
$$

One of our main results in this paper is the following theorem:

Theorem 1.4. Let $T$ be a d-dimensional algebraic torus over a number field $F, X$ a smooth projective toric variety containing $T$ as a Zariski open subset, and $\mathcal{L}$ a metrized line bundle on $X$ (with the metrization introduced in [2]). Assume that the class $[L]$ is contained in the interior of the cone of effective divisors. Then the height zeta function has the following representation:

$$
Z(T, \mathcal{L}, s)=\frac{g(s)}{(s-a(L))^{b(L)}}+h(s)
$$

where $g(s)$ and $h(s)$ are functions holomorphic in the domain $\operatorname{Re}(s) \geq$ $a(L), g(a(L)) \neq 0$, and $b(L)$ is the codimension of the minimal face of $\Lambda_{\mathrm{eff}}(X)$ which contains the class $a(L)[L]+\left[K_{X}\right]$.

Corollary 1.5. We have the following asymptotic formula

$N(T, \mathcal{L}, B)=\frac{g(a(L))}{a(L)(b(L)-1) !} B^{a(L)}(\log B)^{b(L)-1}(1+o(1))$ for $B \rightarrow \infty$.

The paper is organized as follows:

The technical heart of the paper is contained in Section 2, where we investigate analytic properties of some complex valued functions related to convex cones.

In Section 3, we review basic facts from harmonic analysis on the adele group of an algebraic torus.

In Section 4, we recall the terminology from the theory of toric varieties as well as the definition and main properties of heights on toric varieties. 
In Section 5, we give the proof of 1.4. We remark that the most subtle part in the statement of 1.4 is the nonvanishing of the asymptotic constant $g(a(L)) \neq 0$.

\section{TECHNICAL THEOREMS}

Let $I$ and $J$ be two positive integers, $\mathbf{R}[\mathbf{s}, \mathbf{t}]$ (resp. $\mathbf{C}[\mathbf{s}, \mathbf{t}]$ ) the ring of polynomials in $I+J$ variables $s_{1}, \ldots, s_{I}, t_{1}, \ldots, t_{J}$ with coefficients in $\mathbf{R}$ (resp. in $\mathbf{C}$ ) and $\mathbf{C}[[\mathbf{s}, \mathbf{t}]]$ the ring of formal power series in $s_{1}, \ldots, s_{I}, t_{1}, \ldots, t_{J}$ with complex coefficients.

Definition 2.1. Two elements $f(\mathbf{s}, \mathbf{t}), g(\mathbf{s}, \mathbf{t}) \in \mathbf{C}[[\mathbf{s}, \mathbf{t}]]$ will be called coprime, if g.c.d. $(f(\mathbf{s}, \mathbf{t}), g(\mathbf{s}, \mathbf{t}))=1$.

Definition 2.2. Let $f(\mathbf{s}, \mathbf{t})$ be an element of $\mathbf{C}[[\mathbf{s}, \mathbf{t}]]$. By the order of a monomial $s_{1}^{\alpha_{1}} \cdots s_{I}^{\alpha_{I}} t_{1}^{\beta_{1}} \cdots t_{J}^{\beta_{J}}$ we mean the sum of the exponents

$$
\alpha_{1}+\cdots+\alpha_{I}+\beta_{1}+\cdots+\beta_{J} .
$$

By the multiplicity $\mu(f(\mathbf{s}, \mathbf{t}))$ of $f(\mathbf{s}, \mathbf{t})$ at $\mathbf{0}=(0, \ldots, 0)$ we always mean the minimal order of non-zero monomials appearing in the Taylor expansion of $f(\mathbf{s}, \mathbf{t})$ at $\mathbf{0}$.

Definition 2.3. Let $f(\mathbf{s}, \mathbf{t})$ be a meromorphic at $\mathbf{0}$ function. Define the multiplicity $\mu(f(\mathbf{s}, \mathbf{t}))$ of $f(\mathbf{s}, \mathbf{t})$ at $\mathbf{0}$ as

$$
\mu(f(\mathbf{s}, \mathbf{t}))=\mu\left(g_{1}(\mathbf{s}, \mathbf{t})\right)-\mu\left(g_{2}(\mathbf{s}, \mathbf{t})\right)
$$

where $g_{1}(\mathbf{s}, \mathbf{t})$ and $g_{2}(\mathbf{s}, \mathbf{t})$ are two coprime elements in $\mathbf{C}[[\mathbf{s}, \mathbf{t}]]$ such that $f=g_{1} / g_{2}$.

Remark 2.4. It is easy to show that for any two meromorphic at $\mathbf{0}$ functions $f_{1}(\mathbf{s}, \mathbf{t})$ and $f_{2}(\mathbf{s}, \mathbf{t})$, one has

(i) $\mu\left(f_{1} \cdot f_{2}\right)=\mu\left(f_{1}\right)+\mu\left(f_{2}\right)$ (in particular, one can omit "coprime" in Definition 2.3);

(ii) $\mu\left(f_{1}+f_{2}\right) \geq \min \left\{\mu\left(f_{1}\right), \mu\left(f_{2}\right)\right\}$;

(iii) $\mu\left(f_{1}+f_{2}\right)=\mu\left(f_{1}\right)$ if $\mu\left(f_{2}\right)>\mu\left(f_{1}\right)$.

Using the properties 2.4(i)-(ii), one immediately obtains from Definition 2.2 the following:

Proposition 2.5. Let $f_{1}(\mathbf{s}, \mathbf{t}) \in \mathbf{C}[[\mathbf{s}, \mathbf{t}]]$ and $f_{2}(\mathbf{s}) \in \mathbf{C}[[\mathbf{s}]]$ be two analytic at $\mathbf{0}$ functions, $l(\mathbf{s}) \in \mathbf{R}[\mathbf{s}]$ a homogeneous linear function in the variables $s_{1}, \ldots, s_{I}$,

$$
\gamma=\left(\gamma^{I}, \gamma^{J}\right)=\left(\gamma_{1}, \ldots, \gamma_{I}, \gamma_{1}^{\prime}, \ldots, \gamma_{J}^{\prime}\right) \in \mathbf{C}^{I+J}
$$


an arbitrary complex vector with $l\left(\gamma^{I}\right) \neq 0$, and $g(\mathbf{s}, \mathbf{t}):=f_{1}(\mathbf{s}, \mathbf{t}) / f_{2}(\mathbf{s})$. Then the multiplicity of the function

$$
\tilde{g}(\mathbf{s}, \mathbf{t}):=\left.\left(\frac{\partial}{\partial z}\right)^{k} g\left(\mathbf{s}+z \cdot \gamma^{I}, \mathbf{t}+z \cdot \gamma^{J}\right)\right|_{z=-l(\mathbf{s}) / l\left(\gamma^{I}\right)}
$$

at $\mathbf{0}$ is at least $\mu(g)-k$, if

$$
\left.f_{2}\left(\mathbf{s}+z \cdot \gamma^{I}\right)\right|_{z=-l(\mathbf{s}) / l\left(\gamma^{I}\right)}
$$

is not identically zero.

Let $\Gamma \subset \mathbf{Z}^{I+J}$ be a sublattice, $\Gamma_{\mathbf{R}} \subset \mathbf{R}^{I+J}\left(\right.$ resp. $\left.\Gamma_{\mathbf{C}} \subset \mathbf{C}^{I+J}\right)$ the scalar extension of $\Gamma$ to a $\mathbf{R}$-subspace (resp. to a $\mathbf{C}$-subspace). We always assume that $\Gamma_{\mathbf{R}} \cap \mathbf{R}_{>0}^{I+J}=0$ and $\Gamma_{\mathbf{R}} \cap \mathbf{R}^{J}=0$. We set $P_{\mathbf{R}}:=\mathbf{R}^{I+J} / \Gamma_{\mathbf{R}}$ and $P_{\mathbf{C}}:=\mathbf{C}^{I+J} / \Gamma_{\mathbf{C}}$. Let $\pi^{I}$ be the natural projection $\mathbf{C}^{I+J} \rightarrow \mathbf{C}^{I}$. Denote by $\psi$ (resp. by $\psi^{I}$ ) the canonical surjective mapping $\mathbf{C}^{I+J} \rightarrow P_{\mathbf{C}}\left(\right.$ resp. $\left.\mathbf{C}^{I} \rightarrow \mathbf{C}^{I} / \pi^{I}\left(\Gamma_{\mathbf{C}}\right)\right)$.

Definition 2.6. A complex analytic function

$$
f(\mathbf{s}, \mathbf{t})=f\left(s_{1}, \ldots, s_{I}, t_{1}, \ldots, t_{J}\right): U \rightarrow \mathbf{C}
$$

defined on an open subset $U \subset \mathbf{C}^{I+J}$ is said to descend to $P_{\mathbf{C}}$ if $f(\mathbf{u})=$ $f\left(\mathbf{u}^{\prime}\right)$ for all $\mathbf{u}, \mathbf{u}^{\prime} \in U$ with $\mathbf{u}-\mathbf{u}^{\prime} \in \Gamma_{\mathbf{C}}$.

Remark 2.7. By definition, if $f(\mathbf{s}, \mathbf{t})$ descends to $P_{\mathbf{C}}$, then there exists an analytic function $g$ on $\psi(U) \subset P_{\mathbf{C}}$ such that $f=g \circ \psi$. Using Cauchy-Riemann equations, one immediatelly obtains that $f$ descends to $P_{\mathbf{C}}$ if and only if for any vector $\alpha \in \Gamma_{\mathbf{R}}$ and any $\mathbf{u}=\left(u_{1}, \ldots, u_{I+J}\right) \in$ $U$ such that $\mathbf{u}+i \alpha \in U$, one has

$$
f(\mathbf{u}+i \alpha)=f(\mathbf{u}) .
$$

Definition 2.8. An analytic function $f(\mathbf{s}, \mathbf{t})$ in the domain $\operatorname{Re}(\mathbf{s}) \in$ $\mathbf{R}_{>0}^{I}, \operatorname{Re}(\mathbf{t}) \in \mathbf{R}_{>-\delta_{0}}^{J}$ (for some $\delta_{0}>0$ ) is called good with respect to $\Gamma$ and the set of variables $\left\{s_{1}, \ldots, s_{I}\right\}$ if it satisfies the following conditions:

(i) $f(\mathbf{s}, \mathbf{t})$ descends to $P_{\mathbf{C}}$;

(ii) There exist pairwise coprime linear homogeneous polynomials

$$
l_{1}(\mathbf{s}), \ldots, l_{p}(\mathbf{s}) \in \mathbf{R}\left[s_{1}, \ldots, s_{I}\right]
$$

and positive integers $k_{1}, \ldots, k_{p}$ such that for every $j \in\{1, \ldots, p\}$ the linear form $l_{j}(\mathbf{s})$ descends to $P_{\mathbf{C}}, l_{j}(\mathbf{s})$ does not vanish for $\operatorname{Re}(\mathbf{s}) \in \mathbf{R}_{>0}^{I}$, and

$$
q(\mathbf{s}, \mathbf{t})=f(\mathbf{s}, \mathbf{t}) \cdot \prod_{j=1}^{p} l_{j}^{k_{j}}(\mathbf{s})
$$

is analytic at $\mathbf{0 .}$ 
(iii) There exists a nonzero constant $C(f)$ and a homogeneous polynomial $q_{0}(\mathbf{s})$ of degree $\mu(q)$ in variables $s_{1}, \ldots, s_{I}$ such that

$$
q(\mathbf{s}, \mathbf{t})=q_{0}(\mathbf{s})+q_{1}(\mathbf{s}, \mathbf{t})
$$

and

$$
\frac{q_{0}(\mathbf{s})}{\prod_{j=1}^{p} l_{j}^{k_{j}}(\mathbf{s})}=C(f) \cdot \mathcal{X}_{\Lambda(I)}\left(\psi^{I}(\mathbf{s})\right),
$$

where $q_{1}(\mathbf{s}, \mathbf{t})$ is an analytic function at $\mathbf{0}$ with $\mu\left(q_{1}\right)>\mu\left(q_{0}\right)$, both functions $q_{0}, q_{1}$ descend to $P_{\mathbf{C}}$, and $\mathcal{X}_{\Lambda(I)}$ is the $\mathcal{X}_{\text {-function of the cone }}$ $\Lambda(I)=\psi^{I}\left(\mathbf{R}_{\geq 0}^{I}\right) \subset \psi^{I}\left(\mathbf{R}^{I}\right)$ (see Definition 6.1).

Remark 2.9. Let $q(\mathbf{s}, \mathbf{t})$ be an arbitrary analytic at $\mathbf{0}$ function. Collecting terms in the Taylor expansion of $q$, we see that there exists a unique homogeneous polynomial $q_{0}(\mathbf{s}, \mathbf{t})$ and an analytic at $\mathbf{0}$ function $q_{1}(\mathbf{s}, \mathbf{t})$ such that

$$
q(\mathbf{s}, \mathbf{t})=q_{0}(\mathbf{s}, \mathbf{t})+q_{1}(\mathbf{s}, \mathbf{t})
$$

with $\mu(q)=\mu\left(q_{0}\right)<\mu\left(q_{1}\right)$. In particular, the polynomial $q_{0}$ and the function $q_{1}$ in 2.8 are uniquely defined.

Definition 2.10. If $f(\mathbf{s}, \mathbf{t})$ is good with respect to $\Gamma$ and the set of variables $\left\{s_{1}, \ldots, s_{I}\right\}$ as above, then the meromorphic function

$$
\frac{q_{0}(\mathbf{s})}{\prod_{j=1}^{p} l_{j}^{k_{j}}(\mathbf{s})}
$$

will be called the principal part of $f(\mathbf{s}, \mathbf{t})$ at $\mathbf{0}$ and the constant $C(f)$ the principal coefficient of $f(\mathbf{s}, \mathbf{t})$ at $\mathbf{0}$.

Suppose that $\operatorname{dim} \psi^{I}\left(\mathbf{R}^{I}\right) \geq 2$. Let $\gamma=\left(\gamma^{I}, \gamma^{J}\right) \in \mathbf{Z}^{I+J}$ be an element which is not contained in $\Gamma, \tilde{\Gamma}:=\Gamma \oplus \mathbf{Z}<\gamma>$, $\tilde{\Gamma}_{\mathbf{R}}:=$ $\Gamma_{\mathbf{R}} \oplus \mathbf{R}<\gamma>, \tilde{P}_{\mathbf{R}}:=\mathbf{R}^{I+J} / \tilde{\Gamma}_{\mathbf{R}}$ and $\tilde{P}_{\mathbf{C}}:=\mathbf{C}^{I+J} / \tilde{\Gamma}_{\mathbf{C}}$. We assume that $\tilde{\Gamma} \cap \mathbf{R}^{J}=\mathbf{0}$ and $\tilde{\Gamma}_{\mathbf{R}} \cap \mathbf{R}_{>0}^{I+J}=0$. We denote by $\tilde{\psi}$ (resp. by $\tilde{\psi}^{I}$ ) the natural projection $\mathbf{C}^{I+J} \rightarrow \tilde{P}_{\mathbf{C}}\left(\right.$ resp. $\mathbf{C}^{I} \rightarrow \mathbf{C}^{I} / \pi^{I}\left(\tilde{\Gamma}_{\mathbf{C}}\right)$ ).

The following easy statement will be helpful in the sequel:

Proposition 2.11. Let $f(\mathbf{s}, \mathbf{t})$ be an analytic at $\mathbf{0}$ function, $l(\mathbf{s}) \in \mathbf{R}[\mathbf{s}]$ a homogeneous linear function such that $l\left(\gamma^{I}\right) \neq 0$. Assume that $f(\mathbf{s}, \mathbf{t})$ and $l(\mathbf{s})$ descend to $P_{\mathbf{C}}$. Then

$$
\tilde{f}(\mathbf{s}, \mathbf{t}):=f\left(\mathbf{s}-\frac{l(\mathbf{s})}{l\left(\gamma^{I}\right)} \cdot \gamma^{I}, \mathbf{t}-\frac{l(\mathbf{s})}{l\left(\gamma^{I}\right)} \cdot \gamma^{J}\right)
$$

descends to $\tilde{P}_{\mathbf{C}}$. 
Theorem 2.12. Let $f(\mathbf{s}, \mathbf{t})$ be a good function with respect to $\Gamma$ and the set of variables $\left\{s_{1}, \ldots, s_{I}\right\}$ as above,

$$
\Phi(\mathbf{s})=\prod_{j: l_{j}\left(\gamma^{I}\right)=0} l_{j}^{k_{j}}(\mathbf{s})
$$

the product of those linear forms $l_{j}(\mathbf{s})(j \in\{1, \ldots, p\})$ which vanish on $\gamma^{I}$. Assume that the following statements hold:

(i) The integral

$$
\tilde{f}(\mathbf{s}, \mathbf{t}):=\frac{1}{2 \pi i} \int_{\operatorname{Re}(z)=0} f\left(\mathbf{s}+z \cdot \gamma^{I}, \mathbf{t}+z \cdot \gamma^{J}\right) d z, \quad z \in \mathbf{C}
$$

converges absolutely and uniformly to a holomorphic function on any compact in the domain $\operatorname{Re}(\mathbf{s}) \in \mathbf{R}_{>0}^{I}, \operatorname{Re}(\mathbf{t}) \in \mathbf{R}_{>-\delta_{0}}^{J}$;

(ii) There exists $\delta>0$ such that the integral

$$
\frac{1}{2 \pi i} \int_{\operatorname{Re}(z)=\delta} \Phi(\mathbf{s}) \cdot f\left(\mathbf{s}+z \cdot \gamma^{I}, \mathbf{t}+z \cdot \gamma^{J}\right) d z
$$

converges absolutely and uniformly in an open neighborhood of $\mathbf{0}$. Moreover, the multiplicity of the meromorphic function

$$
\tilde{f}_{\delta}(\mathbf{s}, \mathbf{t}):=\frac{1}{2 \pi i} \int_{\operatorname{Re}(z)=\delta} f(\mathbf{s}+z \cdot \gamma) d z
$$

at $\mathbf{0}$ is at least $1-\operatorname{dim} \tilde{\psi}^{I}\left(\mathbf{R}^{I}\right)$;

(iii) For any $\operatorname{Re}(\mathbf{s}) \in \mathbf{R}_{>0}^{I}$ and $\operatorname{Re}(\mathbf{t}) \in \mathbf{R}_{>-\delta_{0}}^{J}$, one has

$$
\lim _{\lambda \rightarrow+\infty}\left(\sup _{0 \leq \operatorname{Re}(z) \leq \delta,|\operatorname{Im}(z)|=\lambda}\left|f\left(\mathbf{s}+z \cdot \gamma^{I}, \mathbf{t}+z \cdot \gamma^{J}\right)\right|\right)=0 .
$$

Then $\tilde{f}(\mathbf{s})$ is a good function with respect to $\tilde{\Gamma}$ and $\left\{s_{1}, \ldots, s_{I}\right\}$, and $C(\tilde{f})=C(f)$.

Proof. By our assumption on $\tilde{\Gamma}, \gamma^{I} \neq 0$. We can assume that $l_{j}\left(\gamma^{I}\right)<0$ for $j=1, \ldots, p_{1}, l_{j}\left(\gamma^{I}\right)=0$ for $j=p_{1}+1, \ldots, p_{2}$, and $l_{j}\left(\gamma^{I}\right)>0$ for $j=p_{2}+1, \ldots, p$. In particular, one has

$$
\Phi(\mathbf{s})=\prod_{j=p_{1}+1}^{p_{2}} l_{j}^{k_{j}}(\mathbf{s}),
$$

where $k_{j}\left(j=p_{1}+1, \ldots, p_{2}\right)$ are some positive integers. Denote by $z_{j}$ the solution of the following linear equation in $z$ :

$$
l_{j}(\mathbf{s})+z l_{j}\left(\gamma^{I}\right)=0, \quad j=1, \ldots, p_{1} .
$$

Let $U$ be the intersection of $\mathbf{R}_{>0}^{I+J}$ with an open neighborhood of $\mathbf{0}$ such that $\Phi(\mathbf{s}) \cdot \tilde{f}_{\delta}(\mathbf{s}, \mathbf{t})$ is analytic for all $(\mathbf{s}, \mathbf{t}) \in U$. By the property (i), both functions $\tilde{f}_{\delta}(\mathbf{s}, \mathbf{t})$ and $\tilde{f}(\mathbf{s}, \mathbf{t})$ are analytic in $U$. Moreover, 
the integral formulas for $\tilde{f}_{\delta}(\mathbf{s}, \mathbf{t})$ and $\tilde{f}(\mathbf{s}, \mathbf{t})$ show that the equalities $\tilde{f}_{\delta}(\mathbf{u}+i y \cdot \gamma)=\tilde{f}_{\delta}(\mathbf{u})$ and $\tilde{f}(\mathbf{u}+i y \cdot \gamma)=\tilde{f}(\mathbf{u})$ hold for any $y \in \mathbf{R}$ and $\mathbf{u}, \mathbf{u}+i y \cdot \gamma \in U$. Therefore, both functions $\tilde{f}_{\delta}(\mathbf{s}, \mathbf{t})$ and $\tilde{f}(\mathbf{s}, \mathbf{t})$ descend to $\tilde{P}_{\mathbf{C}}$ (see Remark 2.7 ). Using the properties (i)-(iii), we can apply the residue theorem and obtain

$$
\tilde{f}(\mathbf{s}, \mathbf{t})-\tilde{f}_{\delta}(\mathbf{s}, \mathbf{t})=\sum_{j=1}^{p_{1}} \operatorname{Res}_{z=z_{j}} f\left(\mathbf{s}+z \cdot \gamma^{I}, \mathbf{t}+z \cdot \gamma^{J}\right)
$$

for $\mathbf{s}, \mathbf{t} \in U$. We denote by $U(\gamma)$ the open subset of $U$ which is defined by the inequalities

$$
\frac{l_{j}(\mathbf{s})}{l_{j}\left(\gamma^{I}\right)} \neq \frac{l_{m}(\mathbf{s})}{l_{m}\left(\gamma^{I}\right)} \text { for all } j \neq m, \quad j, m \in\{1, \ldots, p\} .
$$

The open set $U(\gamma)$ is non-empty, since we assume that $g . c . d .\left(l_{j}, l_{m}\right)=1$ for $j \neq m$. Moreover, for $(\mathbf{s}, \mathbf{t}) \in U(\gamma)$, we have

$\operatorname{Res}_{z=z_{j}} f\left(\mathbf{s}+z \cdot \gamma^{I}, \mathbf{t}+z \cdot \gamma^{J}\right)=$

$$
=\left.\frac{1}{\left(k_{j}-1\right) !}\left(\frac{\partial}{\partial z}\right)^{k_{j}-1} \frac{l_{j}\left(\mathbf{s}+z \cdot \gamma^{I}\right)^{k_{j}} q\left(\mathbf{s}+z \cdot \gamma^{I}, \mathbf{t}+z \cdot \gamma^{J}\right)}{l_{j}^{k_{j}}\left(\gamma^{I}\right) \cdot \prod_{m=1}^{p} l_{m}^{k_{m}}\left(\mathbf{s}+z \cdot \gamma^{I}\right)}\right|_{z=z_{j}},
$$

where

$$
z_{j}=-\frac{l_{j}(\mathbf{s})}{l_{j}\left(\gamma^{I}\right)}\left(j=1, \ldots, p_{1}\right)
$$

Let

$$
f(\mathbf{s}, \mathbf{t}) \cdot \prod_{j=1}^{p} l_{j}^{k_{j}}(\mathbf{s})=q(\mathbf{s}, \mathbf{t})=q_{0}(\mathbf{s})+q_{1}(\mathbf{s}, \mathbf{t})
$$

and

$$
\frac{q_{0}(\mathbf{s})}{\prod_{j=1}^{p} l_{j}^{k_{j}}(\mathbf{s})}=C(f) \cdot \mathcal{X}_{\Lambda(I)}\left(\psi^{I}(\mathbf{s})\right)
$$

where $q_{0}(\mathbf{s})$ is a uniquely determined homogeneous polynomial (see Remark 2.9), $q_{0}(\mathbf{s}, \mathbf{t})$ is an analytic at $\mathbf{0}$ function with $\mu(q)=\mu\left(q_{0}\right)<$ $\mu\left(q_{1}\right)$ and $\mathcal{X}_{\Lambda(I)}\left(\psi^{I}(\mathbf{s})\right)$ is the $\mathcal{X}$-function of the cone $\Lambda(I)=\psi^{I}\left(\mathbf{R}_{\geq 0}^{I}\right)$. We set

$$
R_{0}(\mathbf{s}):=\frac{q_{0}(\mathbf{s})}{\prod_{j=1}^{p} l_{j}^{k_{j}}(\mathbf{s})}, \quad R_{1}(\mathbf{s}, \mathbf{t}):=\frac{q_{1}(\mathbf{s}, \mathbf{t})}{\prod_{j=1}^{p} l_{j}^{k_{j}}(\mathbf{s})} .
$$

Then $\mu(f)=\mu\left(R_{0}\right)<\mu\left(R_{1}\right)$. Moreover, $\mu\left(R_{0}\right)=-\operatorname{dim} \psi^{I}\left(\mathbf{R}^{I}\right)$ (see Prop. 6.2). Define

$$
\tilde{R}_{0}(\mathbf{s}):=\sum_{j=1}^{p_{1}} \operatorname{Res}_{z=z_{j}} R_{0}\left(\mathbf{s}+z \cdot \gamma^{I}\right)
$$


and

$$
\tilde{R}_{1}(\mathbf{s}, \mathbf{t}):=\sum_{j=1}^{p_{1}} \operatorname{Res}_{z=z_{j}} R_{1}\left(\mathbf{s}+z \cdot \gamma^{I}, \mathbf{t}+z \cdot \gamma^{J}\right) .
$$

We claim that

$$
\tilde{R}_{0}(\mathbf{s})=C(f) \cdot \mathcal{X}_{\tilde{\Lambda}(I)}\left(\tilde{\psi}^{I}(\mathbf{s})\right)
$$

where $\mathcal{X}_{\tilde{\Lambda}(I)}\left(\tilde{\psi}^{I}(\mathbf{s})\right)$ is the $\mathcal{X}$-function of the cone $\tilde{\Lambda}(I)=\tilde{\psi}^{I}\left(\mathbf{R}_{\geq 0}^{I}\right)$. Indeed, repeating for $\mathcal{X}_{\Lambda(I)}(\psi(\mathbf{s}))$ the same arguments as for $f(\mathbf{s}, \mathbf{t})$, we obtain

$$
\begin{gathered}
\frac{1}{2 \pi i} \int_{\operatorname{Re}(z)=0} \mathcal{X}_{\Lambda(I)}\left(\psi\left(\mathbf{s}+z \cdot \gamma^{I}\right)\right) d z-\frac{1}{2 \pi i} \int_{\operatorname{Re}(z)=\delta} \mathcal{X}_{\Lambda(I)}\left(\psi\left(\mathbf{s}+z \cdot \gamma^{I}\right)\right) d z \\
=\sum_{j=1}^{k_{1}} \operatorname{Res}_{z=z_{j}} \mathcal{X}_{\Lambda(I)}\left(\psi\left(\mathbf{s}+z_{j} \cdot \gamma^{I}\right)\right) .
\end{gathered}
$$

Moving the contour of integration $\operatorname{Re}(z)=\delta(\delta \rightarrow+\infty)$, by residue theorem, we obtain

$$
\int_{\operatorname{Re}(z)=\delta} \mathcal{X}_{\Lambda(I)}\left(\psi\left(\mathbf{s}+z \cdot \gamma^{I}\right)\right) d z=0 .
$$

On the other hand,

$$
\mathcal{X}_{\tilde{\Lambda}(I)}(\tilde{\psi}(\mathbf{s}))=\frac{1}{2 \pi i} \int_{\operatorname{Re}(z)=0} \mathcal{X}_{\Lambda(I)}\left(\psi\left(\mathbf{s}+z \cdot \gamma^{I}\right)\right) d z
$$

(see Theorem 6.3).

Consider the decomposition of $\tilde{f}$ into the sum:

$$
\tilde{f}(\mathbf{s}, \mathbf{t})=\tilde{f}_{\delta}(\mathbf{s}, \mathbf{t})+\tilde{R}_{0}(\mathbf{s})+\tilde{R}_{1}(\mathbf{s}, \mathbf{t}) .
$$

By our assumption in (ii), $\mu\left(\tilde{f}_{\delta}\right) \geq 1-\operatorname{dim} \tilde{\psi}^{I}\left(\mathbf{R}^{I}\right)$. By Proposition 2.5, we have $\mu\left(\tilde{R}_{1}\right) \geq 1+\mu\left(R_{1}\right) \geq 2+\mu\left(R_{0}\right)=1-\operatorname{dim} \tilde{\psi}^{I}\left(\mathbf{R}^{I}\right)$. Using 2.4(iii), we obtain that $\mu(\tilde{f})=\mu\left(\tilde{R}_{0}\right)=-\operatorname{dim} \tilde{\psi}^{I}\left(\mathbf{R}^{I}\right)$ and $\mu\left(\tilde{f}_{\delta}+\tilde{R}_{1}\right)>\mu(\tilde{f})$.

By 2.11, the linear forms

$$
h_{m, j}(\mathbf{s}):=l_{m}\left(\mathbf{s}+z_{j} \cdot \gamma^{I}\right)=l_{m}(\mathbf{s})-\frac{l_{j}(\mathbf{s})}{l_{j}\left(\gamma^{I}\right)} l_{m}\left(\gamma^{I}\right), \quad\left(j=1, \ldots, p_{1}, m \neq j\right)
$$

and the analytic in the domain $U(\gamma)$ functions

$$
\operatorname{Res}_{z=z_{j}} f\left(\mathbf{s}+z \cdot \gamma^{I}, \mathbf{t}+z \cdot \gamma^{J}\right), \quad j=1, \ldots, p_{1}
$$

and

$$
\operatorname{Res}_{z=z_{j}} R_{0}\left(\mathbf{s}+z \cdot \gamma^{I}\right), j=1, \ldots, p_{1}
$$

descend to $\tilde{P}_{\mathbf{C}}$. For any $j \in\left\{1, \ldots, p_{1}\right\}$, let us denote

$$
Q_{j}(\mathbf{s})=\prod_{m \neq j, m=1}^{p} h_{m, j}^{k_{m}}(\mathbf{s}) .
$$


It is clear that

$$
Q_{j}^{k_{j}}(\mathbf{s}) \cdot \operatorname{Res}_{z=z_{j}} f\left(\mathbf{s}+z \cdot \gamma^{I}, \mathbf{t}+z \cdot \gamma^{J}\right)
$$

and

$$
Q_{j}^{k_{j}}(\mathbf{s}) \cdot \operatorname{Res}_{z=z_{j}} R_{0}\left(\mathbf{s}+z \cdot \gamma^{I}\right)
$$

are analytic at $\mathbf{0}$ and $\Phi(\mathbf{s})$ divides each $Q_{j}(\mathbf{s})$. Hence, we obtain that $\tilde{f}(\mathbf{s}, \mathbf{t}) \prod_{j=1}^{p_{1}} Q_{j}^{k_{j}}(\mathbf{s})=$

$$
=\left(\tilde{f}_{\delta}(\mathbf{s}, \mathbf{t})+\sum_{j=1}^{p_{1}} \operatorname{Res}_{z=z_{j}} f\left(\mathbf{s}+z \cdot \gamma^{I}, \mathbf{t}+z \cdot \gamma^{J}\right)\right) \prod_{j=1}^{p_{1}} Q_{j}^{k_{j}}(\mathbf{s})
$$

and

$$
\tilde{R}_{0}(\mathbf{s}) \prod_{j=1}^{p_{1}} Q_{j}^{k_{j}}(\mathbf{s})=\left(\sum_{j=1}^{p_{1}} \operatorname{Res}_{z=z_{j}} R_{0}\left(\mathbf{s}+z \cdot \gamma^{I}\right)\right) \prod_{j=1}^{p_{1}} Q_{j}^{k_{j}}(\mathbf{s})
$$

are analytic at $\mathbf{0 .}$

Let us define the set $\left\{\tilde{l}_{1}(\mathbf{s}), \ldots, \tilde{l}_{\tilde{p}}(\mathbf{s})\right\}$ as a subset of pairwise coprime elements in the set of homogeneous linear forms $\left\{h_{m, j}(\mathbf{s})\right\}(m \in$ $\left.\{1, \ldots, p\}, j \in\left\{1, \ldots, p_{1}\right\}\right)$ such that there exist positive integers $n_{1}, \ldots, n_{\tilde{p}}$ and a representation of the meromorphic functions $\tilde{f}(\mathbf{s}, \mathbf{t})$ and $\tilde{R}_{0}(\mathrm{~s})$ as quotients

$$
\tilde{f}(\mathbf{s}, \mathbf{t})=\frac{\tilde{q}(\mathbf{s}, \mathbf{t})}{\prod_{j=1}^{\tilde{p}} \tilde{l}_{j}^{n_{j}}(\mathbf{s})}, \quad \tilde{R}_{0}(\mathbf{s})=\frac{\tilde{q}_{0}(\mathbf{s})}{\prod_{j=1}^{\tilde{p}} \tilde{l}_{j}^{n_{j}}(\mathbf{s})},
$$

where $\tilde{q}(\mathbf{s}, \mathbf{t})$ is analytic at $\mathbf{0}, \tilde{q}_{0}(\mathbf{s})$ is a homogeneous polynomial, and none of the forms $\tilde{l}_{1}(\mathbf{s}), \ldots, \tilde{l}_{q}(\mathbf{s})$ vanishes for $(\mathbf{s}, \mathbf{t}) \in \mathbf{R}_{>0}^{I+J}$ (the last property can be achieved, because both functions $\tilde{f}(\mathbf{s}, \mathbf{t})$ and $\tilde{R}_{0}(\mathbf{s}, \mathbf{t})$ are analytic in $U$ and the closure of $U$ is equal to $\mathbf{R}_{\geq 0}^{I+J}$ ).

Define

$$
\tilde{q}_{1}(\mathbf{s}, \mathbf{t})=\left(\tilde{f}_{\delta}(\mathbf{s}, \mathbf{t})+\tilde{R}_{1}(\mathbf{s}, \mathbf{t})\right) \cdot \prod_{j=1}^{\tilde{p}} \tilde{l}_{j}^{n_{j}}(\mathbf{s})
$$

Then

$$
\tilde{q}(\mathbf{s}, \mathbf{t})=\tilde{q}_{0}(\mathbf{s})+\tilde{q}_{1}(\mathbf{s}, \mathbf{t})
$$

where $\tilde{q}_{0}(\mathbf{s}, \mathbf{t})$ is a homogeneous polynomial and $\tilde{q}_{1}(\mathbf{s}, \mathbf{t})$ is an analytic at $\mathbf{0}$ function such that $\mu(\tilde{q})=\mu\left(\tilde{q}_{0}\right)<\mu\left(\tilde{q}_{1}\right)$. Moreover,

$$
\frac{\tilde{q}_{0}(\mathbf{s})}{\prod_{j=1}^{\tilde{p}} \tilde{l}_{j}^{n_{j}}(\mathbf{s})}=C(f) \cdot \mathcal{X}_{\tilde{\Lambda}(I)}\left(\tilde{\psi}^{I}(\mathbf{s})\right)
$$

i.e., $\tilde{f}$ is good. 
Definition 2.13. For any finite dimensional Banach space $V$ over $\mathbf{R}$ we denote by $\|\cdot\|$ a representative in the class of equivalent norms on $V$. For $\mathbf{y}=\left(y_{1}, \ldots, y_{r}\right) \in \mathbf{R}^{r}$ we will set

$$
\|\mathbf{y}\|:=\sum_{j=1}^{r}\left|y_{j}\right| .
$$

The following lemma is elementary:

Lemma 2.14. Let $V=V_{1} \oplus V_{2}$ be a direct sum of finite dimensional vector spaces over $\mathbf{R}, r_{2}$ is the dimension of $V_{2}$, and $r_{2}>0$. Let $f(\mathbf{x})$ be a complex valued function on $V$ satisfying the inequality

$$
|f(\mathbf{x})| \leq \frac{c}{(1+\|\mathbf{x}\|)^{r_{1}+r_{2}+2 \varepsilon}}
$$

for any $\mathbf{x}=\left(\mathbf{x}_{1}, \mathbf{x}_{2}\right) \in V$ and some constants $c, \varepsilon>0$. Let $W \subset V_{2}$ be a locally closed subgroup such that $V_{2} / W$ is compact. Choose any Haar measure $\mathbf{d w}$ on $W$. Then there exists a constant $c^{\prime}>0$ such that we have the estimate

$$
\int_{W}\left|f\left(\mathbf{x}_{1}+\mathbf{w}\right)\right| \mathbf{d} \mathbf{w} \leq \frac{c^{\prime}}{\left(1+\left\|\mathbf{x}_{1}\right\|\right)^{r_{1}+\varepsilon}}
$$

for any $\mathbf{x}_{1} \in V_{1}$.

Theorem 2.15. Let $f(\mathbf{s}, \mathbf{t})$ be an analytic function for $\operatorname{Re}(\mathbf{s}) \in \mathbf{R}_{>0}^{I}$, $\operatorname{Re}(\mathbf{t}) \in \mathbf{R}_{>-\delta_{0}}^{J}$ (for some $\left.\delta_{0}>0\right), \Gamma \subset \mathbf{Z}^{I+J}$ a sublattice of rank $t<I$ with $\Gamma_{\mathbf{R}} \cap \mathbf{R}^{J}=0$ and $\Gamma_{\mathbf{R}} \cap \mathbf{R}_{\geq 0}^{I+J}=0$. Assume that there exist constants $\varepsilon, \varepsilon_{0}>0$ such that the following holds:

(i) The function

$$
g(\mathbf{s}, \mathbf{t})=s_{1} \cdots s_{I} f(\mathbf{s}, \mathbf{t})
$$

is holomorphic in the domain $\operatorname{Re}(\mathbf{s}) \in \mathbf{R}_{>-\varepsilon}^{I}$, $\operatorname{Re}(\mathbf{t}) \in \mathbf{R}_{>-\delta_{0}}^{J}$ and $C(f):=g(\mathbf{0}) \neq 0$;

(ii) For all $\varepsilon_{1}\left(\right.$ with $\left.0<\varepsilon_{1}<\varepsilon\right)$ there exist a constant $C\left(\varepsilon_{1}\right)>0$ and an estimate

$$
\begin{gathered}
\left|f\left(\mathbf{s}+i \mathbf{y}_{I}, \mathbf{t}+i \mathbf{y}_{J}\right)\right| \leq \frac{C\left(\varepsilon_{1}\right)}{(1+\|\mathbf{y}\|)^{t+\varepsilon_{0}}}, \\
\mathbf{y}=\left(\mathbf{y}_{I}, \mathbf{y}_{J}\right), \quad\|\mathbf{y}\|=\left\|\mathbf{y}_{I}\right\|+\left\|\mathbf{y}_{J}\right\|,
\end{gathered}
$$

which holds for all $\mathbf{s}$ such that one of the two inequalities $-\varepsilon<\operatorname{Re}\left(s_{j}\right)<$ $\varepsilon_{1}$ or $\operatorname{Re}\left(s_{j}\right)>\varepsilon_{1}$ is satisfied for every $j=1, \ldots, I$.

Then the integral

$$
\frac{1}{(2 \pi)^{t}} \int_{\Gamma_{\mathbf{R}}} f\left(\mathbf{s}+i \mathbf{y}_{I}, \mathbf{t}+i \mathbf{y}_{J}\right) \mathbf{d} \mathbf{y}
$$

is a good function with respect to $\Gamma$ and the set of variables $\left\{s_{1}, \ldots, s_{I}\right\}$, and $C(f)$ is its principal coefficient. 
Proof. Without loss of generality we can assume that $\Gamma$ is not contained in any of $I$ coordinate hyperplanes $s_{j}=0(j=1, \ldots, I)$, otherwise we reduce the problem to a smaller value of $I$. Therefore, we can choose a basis $\gamma^{1}, \ldots, \gamma^{t}$ of $\Gamma$ such that all first $I$ coordinates of $\gamma^{u}=\left(\gamma_{I}^{u}, \gamma_{J}^{u}\right) \in$ $\mathbf{Z}^{I+J}$ are not equal to 0 for every $u=1, \ldots, t$.

For any non-negative integer $u \leq t$ we define a subgroup $\Gamma^{(u)} \subset \Gamma$ of rank $u$ as follows:

$$
\Gamma^{(0)}=0 ; \quad \Gamma^{(u)}:=\bigoplus_{j=1}^{u} \mathbf{Z}<\gamma^{u}>, u=1, \ldots, t .
$$

We introduce some auxiliary functions

$$
\begin{gathered}
f^{(0)}(\mathbf{s}, \mathbf{t})=f(\mathbf{s}, \mathbf{t}) \\
f^{(u)}(\mathbf{s}, \mathbf{t})=\frac{1}{(2 \pi)^{u}} \int_{\Gamma_{\mathbf{R}}^{(u)}} f\left(\mathbf{s}+i \mathbf{y}_{I}^{(u)}, \mathbf{t}+i \mathbf{y}_{J}^{(u)}\right) \mathbf{d} \mathbf{y}^{(u)}, u=1, \ldots, t,
\end{gathered}
$$

where $\mathbf{d} \mathbf{y}^{(u)}$ is the Lebesgue measure on $\Gamma_{\mathbf{R}}^{(u)}$ normalised by the lattice $\Gamma^{(u)}$. Denote by $P_{\mathbf{C}}^{(u)}=\mathbf{C}^{r} / \Gamma_{\mathbf{C}}^{(u)}$. By the estimate in (ii), $f^{(u)}(\mathbf{s}, \mathbf{t})$ is a holomorphic function in the domain $(\operatorname{Re}(\mathbf{s}), \operatorname{Re}(\mathbf{t})) \in \mathbf{R}_{>0}^{I+J}$ and descends to $P_{\mathbf{C}}^{(u)}$.

We prove by induction that $f^{(u)}(\mathbf{s}, \mathbf{t})$ is good with respect to $\Gamma^{(u)} \subset$ $\mathbf{Z}^{I+J}$ and $\left\{s_{1}, \ldots, s_{I}\right\}$. By (i), $f^{(0)}(\mathbf{s}, \mathbf{t})$ is good. By induction assumption, we know that $f^{(u-1)}(\mathbf{s}, \mathbf{t})$ is good with respect to $\Gamma^{(u-1)}$ and $\left\{s_{1}, \ldots, s_{I}\right\}$. Moreover, we have

$$
f^{(u)}(\mathbf{s}, \mathbf{t})=\frac{1}{(2 \pi i)} \int_{\operatorname{Re}(z)=0} f^{(u-1)}\left(\mathbf{s}+z \cdot \gamma_{I}^{u}, \mathbf{t}+z \cdot \gamma_{J}^{u}\right) d z .
$$

Choose $\delta_{u}>0$ in such a way that for every $j=1, \ldots, I$ one of the following two inequalities is satisfied:

$$
-\varepsilon<\delta_{u} \gamma_{j}^{u}<-\varepsilon_{1}, \text { or } \delta_{u} \gamma_{j}^{u}>\varepsilon_{1}
$$

for some $0<\varepsilon_{1}<\varepsilon$. By (ii), the integral

$$
\begin{aligned}
& f_{\delta}^{(u)}(\mathbf{s}, \mathbf{t})=\frac{1}{(2 \pi i)} \int_{\operatorname{Re}(z)=\delta_{u}} f^{(u-1)}\left(\mathbf{s}+z \cdot \gamma_{I}^{u}, \mathbf{t}+z \cdot \gamma_{J}^{u}\right) d z \\
& =\frac{1}{(2 \pi)^{u}} \int_{\Gamma_{\mathbf{R}}^{(u)}} f\left(\mathbf{s}+\delta_{u} \gamma_{I}^{u}+i \mathbf{y}_{I}^{(u)}, \mathbf{t}+\delta_{u} \gamma_{J}^{u}+i \mathbf{y}_{J}^{(u)}\right) \mathbf{d} \mathbf{y}^{(u)}
\end{aligned}
$$

converges absolutely and uniformly in an open neighborhood of $\mathbf{0}$, i.e. the multiplicity of $f_{\delta}^{(u)}(\mathbf{s}, \mathbf{t})$ is at least $0 \geq 1+\operatorname{rk} \Gamma^{(u)}-I$. Hence it is holomorphic at $\mathbf{0}$ and satisfies assumption (ii) of 2.12. By lemma 2.14, the property 2.12 (iii) holds. Applying theorem 2.12, we conclude that $f^{(u)}(\mathbf{s}, \mathbf{t})$ is a good function with the principal coefficient $g(\mathbf{0})$. 


\section{Fourier ANALYSis ON ALGEBRAIC TORI}

Let $X_{F}$ be an algebraic variety over a number field $F$ and $E / F$ a finite extension of number fields. We shall denote by $X_{E}$ the $E$-variety obtained by base change from $X_{F}$ and by $X(E)$ the set of $E$-rational points of $X_{F}$. Sometimes we omit the subscript in $X_{E}$ if the field is clear from the context.

Let $\mathbf{G}_{m}=\operatorname{Spec}\left(F\left[x, x^{-1}\right]\right)$ be the multiplicative group scheme over $F$. A $d$-dimensional algebraic torus $T$ is a group scheme over $F$ such that over some finite field extension $E / F$ we have $T_{E} \cong\left(\mathbf{G}_{m}\right)^{d}$. We call the minimal $E$ with this property the splitting field of $T$. Denote by $G=\operatorname{Gal}(E / F)$ the Galois group of $E$ over $F$. For every $G$-module $A, A^{G}$ stands for the submodule of elements fixed by $G$. For any field $E$ we denote by $\hat{T}_{E}$ the $G$-module $\operatorname{Hom}\left(T_{E}, \mathbf{G}_{m}\right)$ of $E$-rational characters of $T$. If $E$ is the splitting field of $T$, we put $M:=\hat{T}_{E}$ and $N:=\operatorname{Hom}(M, \mathbf{Z})$ the dual $G$-module. We denote by $t$ the rank of the lattice $M^{G}$.

Let $T$ be an algebraic torus over a number field $F$. Denote by $\operatorname{Val}(F)$ the set of valuations of $F$ and by $\operatorname{Val}_{\infty}(F)$ the set of archimedian valuations. Let $F_{v}$ be the completion of $F$ with respect to $v \in \operatorname{Val}(F), \mathcal{V}$ an extension of $v$ to $E$,

$$
G_{v}:=\operatorname{Gal}\left(E_{\mathcal{V}} / F_{v}\right) \subset \operatorname{Gal}(E / F)
$$

the decomposition group at $v, T\left(F_{v}\right)$ the group of $F_{v}$-rational points of $T$ and $T\left(\mathcal{O}_{v}\right)$ its maximal compact subgroup. We have the canonical embeddings

$$
\pi_{v}: T\left(F_{v}\right) / T\left(\mathcal{O}_{v}\right) \hookrightarrow N^{G_{v}}
$$

for all non-archimedian $v \in \operatorname{Val}(F)$ and

$$
\pi_{v}: T\left(F_{v}\right) / T\left(\mathcal{O}_{v}\right) \hookrightarrow N_{\mathbf{R}}^{G_{v}}
$$

for all $v \in \operatorname{Val}_{\infty}(F)$. Denote by $\bar{x}_{v}$ the image of $x_{v} \in T\left(F_{v}\right)$ in $N^{G_{v}}$ (resp. $N_{\mathbf{R}}^{G_{v}}$ ) under $\pi_{v}$.

Definition 3.1. We call a valuation $v \in \operatorname{Val}(F)$ good, if the mapping $\pi_{v}$ is an isomorphism. We denote by $S$ a finite subset in $\operatorname{Val}(F)$ containing $\operatorname{Val}_{\infty}(F)$ and all valuations $v \in \operatorname{Val}(F)$ which are not good.

Let us recall some basic arithmetic properties of algebraic tori over the ring of adeles $\mathbf{A}_{F}$. Define

$$
T^{1}\left(\mathbf{A}_{F}\right)=\left\{\left.\mathbf{x} \in T\left(\mathbf{A}_{F}\right)\left|\prod_{v \in \operatorname{Val}(F)}\right| m\left(x_{v}\right)\right|_{v}=1, \text { for all } m \in M^{G}\right\} .
$$

Let $\mathbf{K}_{T}=\prod_{v \in \operatorname{Val}(F)} T\left(\mathcal{O}_{v}\right)$ be the maximal compact subgroup of $T\left(\mathbf{A}_{F}\right)$. 
Proposition 3.2. The groups $T\left(\mathbf{A}_{F}\right), T^{1}\left(\mathbf{A}_{F}\right), T(F), \mathbf{K}_{T}$ have the following properties:

(i) $T\left(\mathbf{A}_{F}\right) / T^{1}\left(\mathbf{A}_{F}\right) \cong N_{\mathbf{R}}^{G} \cong \mathbf{R}^{t}$;

(ii) $T^{1}\left(\mathbf{A}_{F}\right) / T(F)$ is compact;

(iii) $T^{1}\left(\mathbf{A}_{F}\right) / T(F) \mathbf{K}_{T}$ is isomorphic to the product a finite group $\mathbf{c l}(T)$, and a connected compact abelian group;

(iv) $w(T)=\mathbf{K}_{T} \cap T(F)$ is a finite abelian group of torsion elements in $T(F)$.

Let $T(\mathcal{O}) \subset T(F)$ be the subgroup of $\mathcal{O}_{F}$-integral points. Then $T(\mathcal{O})$ contains $w(T)$, and $\mathcal{E}_{T}:=T\left(\mathcal{O}_{F}\right) / w(T)$ has a canonical embedding, as a discrete subgroup, into the archimedian logarithmic space

$$
N_{\mathbf{R}, \infty}=\bigoplus_{v \in \operatorname{Val}_{\infty}(F)} N_{\mathbf{R}}^{G_{v}}=\bigoplus_{v \in \operatorname{Val}_{\infty}(F)} T\left(F_{v}\right) / T\left(\mathcal{O}_{v}\right)
$$

Moreover, the image of $\mathcal{E}_{T}$ in $N_{\mathbf{R}, \infty}$ is contained in the $\mathbf{R}$-subspace $N_{\mathbf{R}, \infty}^{1}$ defined as

$$
N_{\mathbf{R}, \infty}^{1}:=\left\{\bar{x} \in N_{\mathbf{R}, \infty} \mid \sum_{v \in \operatorname{Val}_{\infty}(F)} m\left(\bar{x}_{v}\right)=0 \text { for all } m \in M^{G}\right\},
$$

and the quotient $N_{\mathbf{R}, \infty}^{1} / \mathcal{E}_{T}$ is compact.

Definition 3.3. Let $T$ be an algebraic torus over a number field $F$. We define

$$
\mathcal{H}_{T}:=\left(T\left(\mathbf{A}_{F}\right) / T(F)\right)^{*}
$$

as the group of topological characters of $T\left(\mathbf{A}_{F}\right)$ which are trivial on $T(F)$. Define the group $\mathcal{D}_{T}$ as

$$
\mathcal{D}_{T}:=\left(T^{1}\left(\mathbf{A}_{F}\right) / T(F)\right)^{*} .
$$

Define the group $\mathcal{U}_{T}$ as:

$$
\mathcal{U}_{T}:=\left(T^{1}\left(\mathbf{A}_{F}\right) / T(F) \mathbf{K}_{T}\right)^{*} .
$$

We call the characters $\chi \in \mathcal{D}_{T}$ discrete and $\chi \in \mathcal{U}_{T}$ unramified.

Using 3.2 (i), we see that a choice of a splitting of the exact sequence

$$
1 \rightarrow T^{1}\left(\mathbf{A}_{F}\right) \rightarrow T\left(\mathbf{A}_{F}\right) \rightarrow T\left(\mathbf{A}_{F}\right) / T^{1}\left(\mathbf{A}_{F}\right) \rightarrow 1
$$

defines isomorphisms

$$
\begin{aligned}
\mathcal{H}_{T} & \cong M_{\mathbf{R}}^{G} \oplus \mathcal{D}_{T}, \\
N_{\mathbf{R}, \infty} & =N_{\mathbf{R}}^{G} \oplus N_{\mathbf{R}, \infty}^{1},
\end{aligned}
$$

and

where

$$
M_{\mathbf{R}, \infty}=M_{\mathbf{R}}^{G} \oplus M_{\mathbf{R}, \infty}^{1},
$$

$$
M_{\mathbf{R}, \infty}=\bigoplus_{v \in \operatorname{Val}_{\infty}(F)} M_{\mathbf{R}}^{G_{v}}
$$


and $M_{\mathbf{R}, \infty}^{1}$ is the minimal $\mathbf{R}$-subspace in $M_{\mathbf{R}, \infty}$ containing the image of $\mathcal{U}_{T}$ under the canonical mapping

$$
\mathcal{U}_{T} \rightarrow M_{\mathbf{R}, \infty}
$$

From now on we fix such a non-canonical splitting. This allows to consider $\mathcal{U}_{T}$ as a subgroup of $\mathcal{H}_{T}$. By 3.2, we have:

Proposition 3.4. There is an exact sequence

$$
0 \rightarrow \mathbf{c l}^{*}(T) \rightarrow \mathcal{U}_{T} \rightarrow \mathcal{M}_{T} \rightarrow 0
$$

where $\mathcal{M}_{T}$ is the image of the canonical projection of $\mathcal{U}_{T}$ to $M_{\mathbf{R}, \infty}^{1}$ and $\mathbf{c l}^{*}(T)$ is a finite abelian group dual to $\mathbf{c l}(T)$.

We see from 3.4 that a character $\chi \in M_{\mathbf{R}}^{G} \oplus \mathcal{U}_{T}$ is determined by its archimedian component which is an element in $M_{\mathbf{R}, \infty}$ up to a finite choice. Denote by $y(\chi) \in M_{\mathbf{R}}^{G} \oplus \mathcal{M}_{T}$ the image of $\chi \in M_{\mathbf{R}}^{G} \oplus \mathcal{U}_{T}$ in $M_{\mathbf{R}, \infty}$.

For all valuations $v$ we choose Haar measures $d \mu_{v}$ on $T\left(F_{v}\right)$ normalized by

$$
\int_{T\left(\mathcal{O}_{v}\right)} d \mu_{v}=1
$$

We define the canonical measure on the group $T\left(\mathbf{A}_{F}\right)$

$$
\omega=\prod_{v \in \operatorname{Val}(F)} d \mu_{v}
$$

For archimedian valuations the Haar measure $d \mu_{v}$ is the pullback of the Lebesgue measure on $N_{\mathbf{R}}^{G_{v}}$ under the logarithmic map

$$
T\left(F_{v}\right) / T\left(\mathcal{O}_{v}\right) \rightarrow N_{\mathbf{R}}^{G_{v}} .
$$

Let $\mathbf{d x}$ be the Lebesgue measure on $T\left(\mathbf{A}_{F}\right) / T^{1}\left(\mathbf{A}_{F}\right)$. There exists a unique Haar measure $\omega^{1}$ on $T^{1}\left(\mathbf{A}_{F}\right)$ such that $\omega=\omega^{1} \mathbf{d x}$. We define

$$
b(T)=\int_{T^{1}\left(\mathbf{A}_{F}\right) / T(F)} \omega^{1} .
$$

For any $L^{1}$-function $f$ on $T\left(\mathbf{A}_{F}\right)$ and any topological character $\chi$ we denote by $\hat{f}(\chi)$ its global Fourier transform with respect to $\omega$ and by $\hat{f}_{v}\left(\chi_{v}\right)$ the local Fourier transforms. We will use the following version of the Poisson formula:

Theorem 3.5. Let $\mathcal{G}$ be a locally compact abelian group with Haar measure $d g, \mathcal{G}_{0} \subset \mathcal{G}$ a closed subgroup with Haar measure $d g_{0}$. The factor group $\mathcal{G} / \mathcal{G}_{0}$ has a unique Haar measure $d x$ normalized by the condition $d g=d x \cdot d g_{0}$. Let $f: \mathcal{G} \rightarrow \mathbf{C}$ be an $L^{1}$-function on $\mathcal{G}$ and $\hat{f}$ its Fourier transform with respect to $d g$. Suppose that $\hat{f}$ is also an 
$L^{1}$-function on $\mathcal{G}_{0}^{\perp}$, where $\mathcal{G}_{0}^{\perp}$ is the group of topological characters $\chi$ which are trivial on $\mathcal{G}_{0}$. Then

$$
\int_{\mathcal{G}_{0}} f(x) d g_{0}=\int_{\mathcal{G}_{0}^{\perp}} \hat{f}(\chi) d \chi
$$

where $d \chi$ is the orthogonal Haar measure on $\mathcal{G}_{0}^{\perp}$ with respect to the Haar measure $d x$ on $\mathcal{G} / \mathcal{G}_{0}$.

We will apply this formula with $\mathcal{G}=T\left(\mathbf{A}_{F}\right), \mathcal{G}_{0}=T(F), d g=\omega$ and $d g_{0}$ is the discrete measure on $T(F)$. The Haar measure $d \chi$ induces the Lebesgue measure on $M_{\mathbf{R}}^{G}$ normalized by the lattice $M^{G} \subset M_{\mathbf{R}}^{G}$ and the discrete measure on $\mathcal{D}_{T}$.

Definition 3.6. Let $T$ be an algebraic torus over $F$ and $\overline{T(F)}$ the closure of $T(F)$ in $T\left(\mathbf{A}_{F}\right)$ in the direct product topology. Define the obstruction group to weak approximation as

$$
A(T)=T\left(\mathbf{A}_{F}\right) / \overline{T(F)} \text {. }
$$

\section{GeOmetry of toriC VARIETIES}

Definition 4.1. A complete regular $d$-dimensional fan $G$-invariant $\Sigma$ is a finite set of convex rational polyhedral cones in $N_{\mathbf{R}}$ satisfying the following conditions:

(i) every cone $\sigma \in \Sigma$ contains $0 \in N_{\mathbf{R}}$;

(ii) every face $\sigma^{\prime}$ of a cone $\sigma \in \Sigma$ belongs to $\Sigma$;

(iii) the intersection of any two cones in $\Sigma$ is a face of both cones;

(iv) $N_{\mathbf{R}}$ is the union of cones from $\Sigma$;

(v) every cone $\sigma \in \Sigma$ is generated by a part of a Z $\mathbf{Z}$-basis of $N$;

(vi) For any $g \in G$ and any $\sigma \in \Sigma$, one has $g(\sigma) \in \Sigma$.

A complete regular $d$-dimensional fan $\Sigma$ defines a smooth toric variety $X_{\Sigma, E}$ as follows:

$$
X_{\Sigma, E}=\bigcup_{\sigma \in \Sigma} U_{\sigma}=\bigcup_{\sigma \in \Sigma} \operatorname{Spec}(E[M \cap \check{\sigma}])
$$

where $\check{\sigma} \subset M_{\mathbf{R}}$ is the dual to $\sigma$ cone. We can see that $T_{E} \subset U_{\sigma}$ for all $\sigma \in \Sigma$ and that $U_{0}=T$.

Theorem 4.2. [14] Let $\Sigma$ be a complete regular $G$-invariant fan in $N_{\mathbf{R}}$. Assume that the complete toric variety $X_{\Sigma, E}$ defined over the splitting field $E$ by $\Sigma$ is projective. Then there exists a unique complete algebraic variety $X_{\Sigma, F}$ over $F$ such that its base extension to $E$ is isomorphic to $X_{\Sigma, E}$. 
Denote by $\Sigma(j)$ the subset of $j$-dimensional cones in $\Sigma$ and by $N_{\sigma, \mathbf{R}} \subset$ $N_{\mathbf{R}}$ the minimal linear subspace containing $\sigma$. Let $\left\{e_{1}, \ldots, e_{n}\right\}$ be the set of 1-dimensional generators of $\Sigma$. Denote by $P L(\Sigma)$ the lattice of piecewise linear integral functions on $N$. By definition, a function $\varphi \in P L(\Sigma)$ iff $\varphi(N) \subset \mathbf{Z}$ and the restriction of $\varphi$ to every cone $\sigma \in \Sigma$ is a linear function; equivalently, there exist elements $m_{\sigma} \in M$ such that the restriction of $\varphi$ to $\sigma$ is given by $\left\langle\cdot, m_{\sigma}\right\rangle$ where $\langle\cdot, \cdot\rangle$ is induced from the pairing between $N$ and $M$. The $G$-action on $M$ (and $N)$ induces a $G$-action on the free abelian group $P L(\Sigma)$. Let

$$
\Sigma(1)=\Sigma_{1}(1) \cup \ldots \cup \Sigma_{r}(1)
$$

be the decomposition of $\Sigma(1)$ into a union of $G$-orbits. A $G$-invariant piecewise linear function $\varphi \in P L(\Sigma)^{G}$ is determined by the vector $\mathbf{u}=\left(u_{1}, \ldots, u_{r}\right)$, where $u_{i}$ is the value of $\varphi$ on the generator of some 1-dimensional cone in the $G$-orbit $\Sigma_{i}(1),(i=1, \ldots, r)$. It will be convenient for us to consider complex valued piecewise linear functions and to identify $\varphi=\varphi_{\mathbf{u}} \in P L(\Sigma)_{\mathbf{C}}^{G}$ with its complex coordinates $\mathbf{u}=$ $\left(u_{1}, \ldots, u_{r}\right) \in P L(\Sigma)_{\mathbf{C}}^{G}$.

Theorem 4.3. The toric variety $X_{\Sigma}$ has the following properties:

(i) There is a representation of $X_{\Sigma, E}$ as a disjoint union of split algebraic tori $T_{\sigma, E}$ of dimension $\operatorname{dim} T_{\sigma, E}=d-\operatorname{dim} \sigma$. For each $j$ dimensional cone $\sigma \in \Sigma(j)$ we denote by $T_{\sigma, E}$ the kernel of a homomorphism $T_{E} \rightarrow \mathbf{G}_{m, E}^{j}$ defined by a $\mathbf{Z}$-basis of the sublattice $N \cap N_{\sigma, \mathbf{R}}$.

(ii) The closures of $(d-1)$-dimensional tori corresponding to the 1 dimensional cones $\mathbf{R}_{\geq 0} e_{1}, \ldots, \mathbf{R}_{\geq 0} e_{n} \in \Sigma(1)$ define divisors $\bar{T}_{1}, \ldots, \bar{T}_{n}$. We can identify the lattices $P L(\Sigma)=\oplus_{j=1}^{n} \mathbf{Z}\left[\bar{T}_{j}\right]$.

(iii) There is an exact sequence of $G$-modules

$$
0 \rightarrow M \rightarrow P L(\Sigma) \rightarrow \operatorname{Pic}\left(X_{\Sigma, E}\right) \rightarrow 0,
$$

moreover, we have $\operatorname{Pic}\left(X_{\Sigma, F}\right)=\operatorname{Pic}\left(X_{\Sigma, E}\right)^{G}$;

(iv) The cone of effective divisors $\Lambda_{\mathrm{eff}}\left(X_{\Sigma, F}\right) \subset \operatorname{Pic}\left(X_{\Sigma, F}\right)_{\mathbf{R}}$ is generated by the classes of $G$-invariant divisors

$$
D_{j}=\sum_{\mathbf{R}_{\geq 0} e_{i} \in \Sigma_{j}(1)} \bar{T}_{i}
$$

(v) The class of the anticanonical divisor $-\left[K_{\Sigma}\right]$ is given by the class of the $G$-invariant divisor

$$
-\left[K_{\Sigma}\right]=\left[D_{1}+\ldots+D_{r}\right] .
$$

Remark 4.4. We note that for toric varieties we have $\operatorname{Pic}\left(X_{\Sigma, F}\right)=$ $N S\left(X_{\Sigma, F}\right)$. 
Definition 4.5. Let $\varphi \in P L(\Sigma)_{\mathrm{C}}^{G}$ be a complex valued piecewise linear function. Let $v \in \operatorname{Val}(F)$ be a non-archimedian valuation. Denote by $q_{v}$ the order of the residue field of $F_{v}$. For $x_{v} \in T\left(F_{v}\right)$ we define the complex local height function

$$
H_{\Sigma, v}\left(x_{v}, \varphi\right)=e^{\varphi\left(\bar{x}_{v}\right) \log q_{v}} .
$$

Let $v$ be an archimedian valuation. The complex local height function is defined as

$$
H_{\Sigma, v}\left(x_{v}, \varphi\right)=e^{\varphi\left(\bar{x}_{v}\right)}
$$

Remark 4.6. This provides a piecewise smooth metrization of line bundles on the toric variety $X_{\Sigma}$. One can show that this metrization is, in a sense, "canonical". Namely, an algebraic torus admits a morphism to itself (n-th power morphism), which extends to a compactification. Using the construction of Tate one can obtain a metrization on a line bundle by a limiting process. This metrization coincides with ours.

Definition 4.7. Let $x \in T(F) \subset X_{\Sigma}(F)$ be a rational point. The global height function is defined by

$$
H_{\Sigma}(x, \varphi)=\prod_{v} H_{\Sigma, v}\left(x_{v}, \varphi\right) .
$$

By the product formula, the function $H_{\Sigma}(x, \varphi)$ as a function on $T(F)$ descends to the complexified Picard group $\operatorname{Pic}\left(X_{\Sigma}\right)_{\mathbf{C}}$. Moreover, we have the following

Proposition 4.8. [2] Let $X_{\Sigma}$ be an smooth projective toric variety. For all $x \in T(F) \subset X_{\Sigma}(F)$ the function $H_{\Sigma}(t, \varphi)$ coincides with a classical height corresponding to some metrization of the line bundle $L$ represented by a piecewise linear function $\varphi \in P L(\Sigma)^{G}$.

Let $X_{\Sigma}$ be a toric variety and $H_{\Sigma}$ the height pairing. Clearly, it extends to a pairing $T\left(\mathbf{A}_{F}\right) \times P L(\Sigma)_{\mathbf{C}}^{G} \rightarrow \mathbf{C}$. Moreover, it is invariant under the maximal compact subgroup $\mathbf{K}_{T}=\prod_{v \in \operatorname{Val}(F)} T\left(\mathcal{O}_{v}\right)$. Therefore, its Fourier transform $\hat{H}_{\Sigma}(\chi,-\mathbf{s})$ equals zero for characters $\chi \in \mathcal{H}_{T}$ which are non-trivial on $\mathbf{K}_{T}$.

By 4.3, we have an exact sequence of $\mathbf{Z}$-modules

$$
0 \rightarrow M^{G} \rightarrow P L(\Sigma)^{G} \rightarrow \operatorname{Pic}\left(X_{\Sigma, F}\right) \rightarrow H^{1}(G, M) \rightarrow 0 .
$$

It induces a surjective map of tori $a: \prod_{j=1}^{r} R_{F_{j} / F}\left(\mathbf{G}_{m}\right) \rightarrow T$ and a surjective homomorphism

$$
a: \prod_{h j=1}^{r} \mathbf{G}_{m}\left(\mathbf{A}_{F_{j}}\right) / \mathbf{G}_{m}\left(F_{j}\right) \rightarrow T\left(\mathbf{A}_{F}\right) / T(F)
$$


Every character $\chi \in \mathcal{H}_{T}$ defines $r$ Hecke characters $\chi_{1}, \ldots, \chi_{r}$ of the groups $\mathbf{G}_{m}\left(\mathbf{A}_{F, j}\right) / \mathbf{G}_{m}\left(F_{j}\right)$ by $\chi \circ a$. It is known [6], that $\operatorname{Coker}(a)$ is isomorphic to the obstruction group to weak approximation $A(T)$ (see 3.6). Similarly, every local character $\chi_{v}$ defines local characters $\chi_{1, v}, \ldots \chi_{r, v}$. If $\chi$ is trivial on $\mathbf{K}_{T}$ then all $\chi_{j}$ are trivial on the maximal compact subgroups in in $\mathbf{G}_{m}\left(\mathbf{A}_{F_{j}}\right)$, in other words, all $\chi_{j}$ are unramified. Their local components for all valuations are given by

$$
\begin{gathered}
\chi_{j, v}: \mathbf{G}_{m}\left(F_{j, v}\right) / \mathbf{G}_{m}\left(\mathcal{O}_{j, v}\right) \rightarrow \mathbf{C}^{*} \\
\chi_{j, v}\left(x_{v}\right)=\left|x_{v}\right|_{v}^{i y_{j, v}}
\end{gathered}
$$

for some real $y_{j, v}$.

In the remaining part of this section we recall some estimates which will be used it the study of analytic properties of the height zeta function (see [2]).

Let us consider a Hecke character $\chi \in\left(\mathbf{G}_{m}\left(\mathbf{A}_{F}\right) / \mathbf{G}_{m}(F)\right)^{*}$ and the corresponding Hecke $L$-function $L(\chi, u)$. The following estimate can be proved using the Phragmen-Lindelöf principle [12].

Proposition 4.9. For all $\varepsilon>0$ there exists a constant $c_{1}(\varepsilon)$ such that for all unramified $\chi$ and all $u$ with $\operatorname{Re}(u)>1+\varepsilon$

$$
|L(\chi, u)|<c_{1}(\varepsilon) .
$$

For all $\varepsilon>0$ there exists $a \delta>0$ such that for all unramified $\chi$ and all $u$ with $\operatorname{Re}(u)$ contained in any compact $\mathbf{K}$ in the domain $0<\mid \operatorname{Re}(u)-$ $1 \mid<\delta$ there exists a constant $c(\mathbf{K}, \varepsilon)$ depending only on $\mathbf{K}$ and $\varepsilon$ such that

$$
|L(\chi, u)| \leq c(\mathbf{K}, \varepsilon)(1+|\operatorname{Im}(u)|+\|y(\chi)\|)^{\varepsilon} .
$$

Let $T$ be an algebraic torus and $\chi \in \mathcal{U}_{T}$ an unramified character. Denote by $\chi_{v}$ its local components and by $\chi_{1}, \ldots, \chi_{r}$ the induced unramified Hecke characters of $\mathbf{G}_{m}\left(\mathbf{A}_{F_{j}}\right)$.

Definition 4.10. Define

$$
\zeta_{f i n}(\chi,-\mathbf{u}):=\frac{\prod_{v \notin \operatorname{Val}_{\infty}(F)} \hat{H}_{\Sigma, v}\left(\chi_{v},-\mathbf{u}\right)}{\prod_{j=1}^{r} L_{F_{j}}\left(\chi_{j}, u_{j}\right)},
$$

where for every field $F_{j}$ we denoted by $L_{F_{j}}\left(\chi_{j}, u\right)$ the standard Hecke $L$-function of $F_{j}$. For any $\chi \in \mathcal{D}_{T}$ we define

$$
\zeta_{\infty}(\chi, \mathbf{u}):=\zeta_{f i n}(\chi,-\mathbf{u}) \cdot \prod_{v \in \operatorname{Val}_{\infty}(F)} \hat{H}_{\Sigma, v}(\chi,-\mathbf{u}) .
$$


Proposition 4.11. [2] For every $\delta_{0}>0$ there exist constants $0<$ $c_{1}<c_{2}$ such that for any $\mathbf{u}$ with $\operatorname{Re}(\mathbf{u}) \in \mathbf{R}_{>1 / 2+\delta_{0}}^{r}$ and any $\chi \in \mathcal{U}_{T}$ we have

$$
c_{1}<\left|\zeta_{f i n}(\chi,-\mathbf{u})\right|<c_{2} .
$$

Proposition 4.12. [2] Let $\chi \in \mathcal{U}_{T}$ be an unramified character and $y(\chi)$ its image in $M_{\mathbf{R}, \infty}$. For all $\delta_{0}>0$ there exists a constant $c\left(\delta_{0}\right)$ such that for any $\mathbf{u}$ in the domain $\operatorname{Re}(\mathbf{u}) \in \mathbf{R}_{>1 / 2+\delta_{0}}^{r}$ we have the following estimate

$$
\left|\prod_{v \in \operatorname{Val}_{\infty}(F)} \hat{H}_{\Sigma, v}(\chi,-\mathbf{u})\right| \leq \frac{c\left(\delta_{0}\right)}{(1+\|y(\chi)\|+\|\operatorname{Im}(\mathbf{u})\|)^{\rho+t+1}},
$$

where $\rho+t$ is the dimension of the real vector space $M_{\mathbf{R}, \infty}$.

Corollary 4.13. For any $\delta_{0}>0$, there exists a constant $c\left(\delta_{0}\right)$ such that for any $\chi \in \mathcal{U}_{T}$ and any $\mathbf{u}$ in the domain $\operatorname{Re}(\mathbf{u}) \in \mathbf{R}_{>1 / 2+\delta_{0}}^{r}$ we have the following estimate:

$$
\left|\zeta_{\infty}(\chi, \mathbf{u})\right| \leq \frac{c\left(\delta_{0}\right)}{(1+\|y(\chi)\|+\|\operatorname{Im}(\mathbf{u})\|)^{\rho+t+1}}
$$

\section{AnAlytic properties of height zeta Functions}

Definition 5.1. Let $X_{\Sigma}$ be a smooth projective toric variety. Let $\varphi=$ $\varphi_{\mathbf{u}} \in P L(\Sigma)_{\mathbf{C}}^{G}$ be a complexified piecewise linear function. Let $Y \subset X_{\Sigma}$ be a locally closed subset. The height zeta function with respect to $Y$ is defined as

$$
Z_{\Sigma}(Y, \mathbf{u})=\sum_{x \in Y(F)} H_{\Sigma}(x,-\mathbf{u})
$$

Let us formulate the first main result.

Theorem 5.2. [2] The height zeta function $Z_{\Sigma}(T, \mathbf{u})$ as a function on $P L(\Sigma)_{\mathbf{C}}^{G}$ is holomorphic for $\operatorname{Re}(\mathbf{u}) \in \mathbf{R}_{>1}^{r}$. Moreover, it descends to $\operatorname{Pic}\left(X_{\Sigma}\right)_{\mathbf{C}}$ and is holomorphic for $\operatorname{Re}(\mathbf{u})$ contained in the open cone $\Lambda_{\text {eff }}^{\circ}\left(X_{\Sigma}\right)+\left[K_{\Sigma}\right]$.

Theorem 5.3. (Poisson formula) $[2,3]$ For all $\mathbf{u}$ with $\operatorname{Re}(\mathbf{s}) \in \mathbf{R}_{>1}^{r}$ we have the following formula:

$$
Z_{\Sigma}(T, \mathbf{u})=\frac{1}{(2 \pi)^{t} b(T)} \int_{\mathcal{H}_{T}} \hat{H}_{\Sigma}(\chi,-\mathbf{u}) d \chi
$$

The integral converges absolutely and uniformly to a holomorphic function in $\mathbf{u}$ in any compact in the domain $\operatorname{Re}(\mathbf{u}) \in \mathbf{R}_{>1}^{r}$. 
Let $\mathcal{L}$ be a line bundle on $X_{\Sigma}$ metrized as above, such that its class $[L]$ is contained in the interior of the cone of effective divisors $\Lambda_{\text {eff }}\left(X_{\Sigma}\right) \subset$ $\operatorname{Pic}\left(X_{\Sigma}\right)$. We have defined $a(L)$ as

$$
a(L):=\inf \left\{a \in \mathbf{R} \mid a[L]+\left[K_{\Sigma}\right] \in \Lambda_{\text {eff }}\left(X_{\Sigma}\right)\right\} .
$$

By our assumptions, we have $a(L)>0$, since $-\left[K_{\Sigma}\right] \in \Lambda_{\text {eff }}^{\circ}\left(X_{\Sigma}\right)$. Denote by $\Lambda(L)$ the face of maximal codimension of the cone $\Lambda_{\mathrm{eff}}\left(X_{\Sigma}\right)$ which contains $a(L)[L]+\left[K_{\Sigma}\right]$.

Let $J(L) \subset[1, \ldots, r]$ be the set of indices such that $\left[D_{j}\right] \in \Lambda(L)$ for $j \in$ $J(L)$ and $I(L)=[1, \ldots, r] \backslash J(L)$. We set $I=|I(L)|$ and $J=|J(L)|=$ $r-I$. Without loss of generality, we assume that $I(L)=\{1, \ldots, I\}$ and $J(L)=\{I+1, \ldots, r\}$. Since $a(L)[L]+\left[K_{\Sigma}\right]$ is an interior point of $\Lambda(L)$ it follows that there exists a representation

$$
a(L)[L]+\left[K_{\Sigma}\right]=\sum_{j \in J(L)} \lambda_{j}\left[D_{j}\right]
$$

where $\lambda_{j} \in \mathbf{Q}_{>0}$. Therefore,

$$
[L]=\sum_{j \in J(L)} \frac{\lambda_{j}+1}{a(L)}\left[D_{j}\right]+\sum_{i \in I(L)} \frac{1}{a(L)}\left[D_{i}\right] .
$$

Fix these $\lambda_{j}$ and choose $\varepsilon>0$ such that $2 \varepsilon<\min _{j \in J(L)} \lambda_{j}$. We denote by $\varphi_{L}$ the piecewise linear function from $P L(\Sigma)_{\mathbf{R}}^{G}$ such that $a(L) \varphi_{L}\left(e_{i}\right)=1$ for $i=1, \ldots, I$ and $a(L) \varphi_{L}\left(e_{j}\right)=1+\lambda_{j}$ for $j \in J(L)$. Here $e_{i}$ are generators of one-dimensional cones $\mathbf{R}_{\geq 0} e_{i}$ in the $G$-orbits $\Sigma_{i}(1)$

We introduce the lattice

$$
M_{J}=\left\{m \in M \mid<e, m>=0 \text { for } \mathbf{R}_{\geq 0} e \in \cup_{i=1}^{I} \Sigma_{i}(1)\right\} .
$$

Define $M_{I} \cong M / M_{J}$. The following diagram is commutative

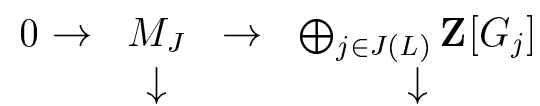

$$
\begin{aligned}
& \begin{array}{c}
0 \rightarrow \underset{\downarrow}{M} \\
\end{array} \quad \rightarrow \quad \bigoplus_{i=1}^{r} \mathbf{Z}\left[G_{i}\right] \\
& 0 \rightarrow M_{I} \rightarrow \bigoplus_{i \in I(L)} \mathbf{Z}\left[G_{i}\right] .
\end{aligned}
$$

The exact sequence of $G$-modules

$$
0 \rightarrow M_{J} \rightarrow M \rightarrow M_{I} \rightarrow 0
$$

induces the exact sequence of algebraic tori

$$
1 \rightarrow T_{I} \rightarrow T \rightarrow T_{J} \rightarrow 1
$$

It will be convenient to introduce new coordinates $\mathbf{s}=\left(s_{i}\right)_{i \in I(L)}, \mathbf{t}=$ $\left(t_{j}\right)_{j \in J(L)}$ on $P L(\Sigma)_{\mathbf{C}}^{G}$, where $s_{i}=u_{i}-1(i=1, \ldots, I), t_{j}=u_{I+j}-1+\varepsilon$ $(j=1, \ldots, J)$. We shall write $(\mathbf{s}, \mathbf{t})=\left(s_{1}, \ldots, s_{I}, t_{1}, \ldots, t_{J}\right)$. 
Theorem 5.4. The height zeta function $Z_{\Sigma}(T, \mathbf{s}, \mathbf{t})$ is good with respect to the lattice $M_{I}^{G}$ and variables $\left\{s_{1}, \ldots, s_{I}\right\}$ in the domain $\operatorname{Re}(\mathbf{s}) \in \mathbf{R}_{>0}^{I}$, $\operatorname{Re}(\mathbf{t}) \in \mathbf{R}_{>-\delta_{0}}^{J}$ for some positive $\delta_{0}<\varepsilon$.

Proof. Recall that $Z_{\Sigma}(T, \mathbf{u})$ has the following integral representation in the domain $\operatorname{Re}(\mathbf{u}) \in \mathbf{R}_{>1}^{r}(5.3)$ :

$$
Z_{\Sigma}(T, \mathbf{u})=\frac{1}{(2 \pi)^{t} b(T)} \int_{\mathcal{H}_{T}} \hat{H}_{\Sigma}(\chi,-\mathbf{u}) d \chi
$$

Using the explicit computation of the Fourier transform of local height functions and the absolute convergence of the integral in the domain $\operatorname{Re}(\mathbf{u}) \in \mathbf{R}_{>1}^{r}$, we have

$$
Z_{\Sigma}(T, \mathbf{u})=\frac{1}{(2 \pi)^{t} b(T)} \int_{M_{\mathbf{R}}^{G}} \mathbf{d y}\left(\sum_{\chi \in \mathcal{U}_{T}} \hat{H}_{\Sigma}(\chi,-\mathbf{u}+i \mathbf{y})\right)
$$

because the local height functions are invariant under the maximal compact subgroups $T\left(\mathcal{O}_{v}\right) \subset T\left(F_{v}\right)$ and $\hat{H}_{\Sigma}(\chi,-\mathbf{u})=0$ for all $\chi$ which are not trivial on the maximal compact subgroup $\mathbf{K}_{T}$. By 4.10 , we have:

$$
\hat{H}_{\Sigma}(\chi,-\mathbf{u})=\prod_{j=1}^{r} L\left(\chi_{j}, u_{j}\right) \times \zeta_{\infty}(\chi, \mathbf{u})
$$

where $\chi_{1}, \ldots, \chi_{r}$ are unramified Hecke characters of $\mathbf{G}_{m}^{1}\left(\mathbf{A}_{F_{j}}\right)$ induced from a character $\chi \in \mathcal{U}_{T}$, and $\zeta_{\infty}(\chi, \mathbf{u})$ is a function in $\mathbf{u}$ which is holomorphic in the domain $\operatorname{Re}(\mathbf{u}) \in \mathbf{R}_{>1 / 2+\delta_{0}}^{r}$ (for all $\delta_{0}>0$ ).

We have

$$
Z_{\Sigma}(T, \mathbf{s}, \mathbf{t})=\frac{1}{(2 \pi)^{t} b(T)} \int_{M_{I, \mathbf{R}}^{G}} f_{\Sigma}\left(\mathbf{s}-i \mathbf{y}_{I}, \mathbf{t}-i \mathbf{y}_{J}\right) \mathbf{d} \mathbf{y}^{I}
$$

where

$$
\begin{gathered}
f_{\Sigma}(\mathbf{s}, \mathbf{t}):=\sum_{\chi \in \mathcal{U}_{T}} \prod_{i \in I(L)} L\left(\chi_{i}, s_{i}+1\right) \times b_{\Sigma}(\chi, \mathbf{s}, \mathbf{t}) \\
b_{\Sigma}(\chi, \mathbf{s}, \mathbf{t})= \\
\left.=\int_{M_{J, \mathbf{R}}^{G}} \prod_{j \in J(L)} L_{F_{j}}\left(\chi_{j}, t_{j}+1+\varepsilon-i y_{j}\right) \times \zeta_{\infty}\left(\chi, \mathbf{s}-i \mathbf{y}_{I}, \mathbf{t}-i \mathbf{y}_{J}\right)\right) \mathbf{d y}^{J},
\end{gathered}
$$

$\mathbf{d} \mathbf{y}^{I}$ is the Lebesgue measure on $M_{I, \mathbf{R}}^{G}$ and $\mathbf{d y}{ }^{J}$ the Lebesgue measure on $M_{J, \mathbf{R}}^{G}$.

Using the estimates $2.14,4.12,4.9,4.13$, we see that the sums and integrals above converge absolutely and uniformly to an analytic function in any compact in the domain $\operatorname{Re}(\mathbf{s}) \in \mathbf{R}_{>0}^{I}$ and $\operatorname{Re}(\mathbf{t}) \in \mathbf{R}_{>-\delta_{0}}^{J}$ for some $\delta_{0}>0\left(\delta_{0}<\varepsilon\right)$. Now the fact that the function $Z_{\Sigma}(T, \mathbf{s}, \mathbf{t})$ is good with respect to the lattice $M_{I}^{G} \subset \mathbf{Z}^{I}$ and the variables $\left(s_{1}, \ldots, s_{I}\right)$ follows from 2.15 and the following statement: 


\section{Theorem 5.5.}

$$
\lim _{\mathbf{s} \rightarrow \mathbf{0}} s_{1} \cdots s_{I} f_{\Sigma}(\mathbf{s}, \mathbf{0})
$$

exists and is not equal to zero.

We divide the proof of Theorem 5.5 into a sequence of lemmas:

Lemma 5.6. Let $\mathcal{U}_{T}(I)$ be the subgroup of $\mathcal{U}_{T}$ consisting of characters $\chi \in \mathcal{U}_{T}$ such that the corresponding Hecke characters $\chi_{i}(i=1, \ldots, I)$ are trivial. Denote

$$
f_{\Sigma}^{I}(\mathbf{s}, \mathbf{t})=\sum_{\chi \in \mathcal{U}_{T}(I)} \prod_{i \in I(L)} L_{F_{i}}\left(\chi_{i}, s_{i}+1\right) b_{\Sigma}(\chi, \mathbf{s}, \mathbf{t}) .
$$

Then

$$
\lim _{\mathbf{s} \rightarrow \mathbf{0}} s_{1} \cdots s_{I} f_{\Sigma}(\mathbf{s}, \mathbf{0})=\lim _{\mathbf{s} \rightarrow \mathbf{0}} s_{1} \cdots s_{I} f_{\Sigma}^{I}(\mathbf{s}, \mathbf{0}) .
$$

Lemma 5.7. (Poisson formula) For $\operatorname{Re}(\mathbf{s}, \mathbf{t}) \in \mathbf{R}_{>0}^{I} \times \mathbf{R}_{>-\delta_{0}}^{J}$, one has:

$$
f_{\Sigma}^{I}(\mathbf{s}, \mathbf{t})=\int_{\mathcal{A}} H_{\Sigma}(x,(-\mathbf{s},-\mathbf{t})) d \alpha,
$$

where the subgroup $\mathcal{A} \subset T\left(\mathbf{A}_{F}\right)$ is defined as

$$
\mathcal{A}:=T(F) \overline{T_{I}(F)} \text {. }
$$

Proof. By definition of $f_{\Sigma}^{I}(\mathbf{s}, \mathbf{t})$, we conclude that this function equals to the integral of the Fourier transform of the adelic height function over the subgroup of characters $\chi$ of $T\left(\mathbf{A}_{F}\right)$ which are trivial on $T(F)$ and such that the induced Hecke characters $\chi_{i}$ are trivial for $i \in I(L)$. It follows from the diagram

$$
\begin{aligned}
& \prod_{i \in I(L)} \mathbf{G}_{m}\left(\mathbf{A}_{F_{i}}\right) / \mathbf{G}_{m}\left(F_{i}\right) \rightarrow T_{I}\left(\mathbf{A}_{F}\right) / T_{I}(F) \rightarrow A\left(T_{I}\right) \rightarrow 0 \\
& \downarrow \quad \downarrow \quad \downarrow \\
& \prod_{i=1}^{r} \mathbf{G}_{m}\left(\mathbf{A}_{F_{i}}\right) / \mathbf{G}_{m}\left(F_{j}\right) \rightarrow T\left(\mathbf{A}_{F}\right) / T(F) \rightarrow A(T) \rightarrow 0 \\
& \prod_{j \in J(L)} \mathbf{G}_{m}\left({\stackrel{\downarrow}{\mathbf{A}_{F}}}\right) / \mathbf{G}_{m}\left(F_{j}\right) \rightarrow T_{J}\left(\mathbf{A}_{F}\right) / T_{J}(F) \rightarrow \stackrel{\downarrow}{ } \rightarrow \stackrel{\left(T_{J}\right)}{\rightarrow} \rightarrow 0 .
\end{aligned}
$$

that the common kernel of all such characters is $T(F) \overline{T_{I}(F)}$ (here we used the isomorphism $\left.A\left(T_{I}\right)=T_{I}\left(\mathbf{A}_{F}\right) / \overline{T_{I}(F)}\right)$. The proof of the absolute convergence of the integral over $\mathcal{A}$ in the domain $\operatorname{Re}(\mathbf{s}, \mathbf{t}) \in$ $\mathbf{R}_{>0}^{I} \times \mathbf{R}_{>-\delta_{0}}^{J}$ is analogous to the proof of theorem 4.2 in [3].

Lemma 5.8. The function

$$
s_{1} \cdots s_{I} f_{\Sigma}^{I}(\mathbf{s}, \mathbf{t})
$$

extends to an analytic function in the domain $\operatorname{Re}(\mathbf{s}, \mathbf{t}) \in \mathbf{R}_{>-\delta_{0}}^{r}$. 
Proof. The proof is similar to the proof of theorem 4.2 in [3]. The integral

$$
\int_{\mathcal{A}} H_{\Sigma}(x,(-\mathbf{s},-\mathbf{t})) d \alpha
$$

can be estimated from above by an Euler product which is absolutely convergent in the domain $\operatorname{Re}(\mathbf{s}, \mathbf{t}) \in \mathbf{R}_{>-\delta_{0}}^{r}$ times a product of zeta functions $\prod_{i=1}^{I} \zeta_{F_{i}}\left(s_{i}+1\right)$.

For $(\mathbf{s}, \mathbf{t}) \in \mathbf{R}^{r}$ the function $H_{\Sigma}(x,(-\mathbf{s},-\mathbf{t}))$ has values in positive real numbers. Therefore, to prove the non-vanishing of the constant, it suffices to show the following:

Lemma 5.9. The value of

$$
s_{1} \cdots s_{I} \int_{\overline{T_{I}(F)}} H_{\Sigma}(x,(-\mathbf{s},-\mathbf{t})) d \alpha_{I}
$$

at $(\mathbf{0}, \mathbf{0})$ is positive. Here $d \alpha_{I}$ is the induced Haar measure on $\overline{T_{I}(F)}$

Proof. For some finite subset $S \subset \operatorname{Val}(F)$, we can split the group $\overline{T_{I}(F)}$ into the direct product

$$
{\overline{T_{I}(F)_{S}}} \times T_{I}\left(\mathbf{A}_{F, S}\right),
$$

where ${\overline{T_{I}(F)}}_{S}$ is the image of $\overline{T_{I}(F)}$ in the finite product $\prod_{v \in S} T_{I}\left(F_{v}\right)$ and

$$
T_{I}\left(\mathbf{A}_{F, S}\right)=T_{I}\left(\mathbf{A}_{F}\right) \cap \prod_{v \notin S} T_{I}\left(F_{v}\right) .
$$

Hence,

$$
\begin{gathered}
\int \frac{\int_{\overline{T_{I}(F)}}}{} H_{\Sigma}(x,(-\mathbf{s},-\mathbf{t})) d \alpha_{I}= \\
=\int \frac{T_{T_{I}(F)_{S}}}{} H_{\Sigma}(x,(-\mathbf{s},-\mathbf{t})) d \alpha_{S} \times \prod_{v \notin S} \int_{T_{I}\left(F_{v}\right)} H_{\Sigma, v}(x,(-\mathbf{s},-\mathbf{t})) d \alpha_{v} .
\end{gathered}
$$

Here we denoted by $d \alpha_{S}$ and $d \alpha_{v}$ the Haar measures induced from $d \alpha_{I}$. We claim that

$$
\prod_{i=1}^{I} \zeta_{F_{i}}^{-1}\left(s_{i}+1\right) \prod_{v \notin S} \int_{T_{I}\left(F_{v}\right)} H_{\Sigma, v}(x,(-\mathbf{s},-\mathbf{t})) d \alpha_{v} .
$$

is an absolutely convergent Euler product for $\operatorname{Re}(\mathbf{s}, \mathbf{t}) \in \mathbf{R}_{>-\delta_{0}}^{r}$. This statement follows from the explicit calculation of the local integrals (see 5.10).

Lemma 5.10. For all good valuations $v \notin S$, the local integral

$$
\int_{T_{I}\left(F_{v}\right)} H_{\Sigma, v}(x,(-\mathbf{s},-\mathbf{t})) d \alpha_{v}=\prod_{i=1}^{I} \prod_{\mathcal{V} \mid v} \zeta_{F_{i}, \mathcal{V}}\left(s_{i}+1\right)\left(1+o\left(q_{v}^{-1-\varepsilon_{0}}\right)\right)
$$

for some $\varepsilon_{0}>0$ and all $\operatorname{Re}(\mathbf{s}, \mathbf{t}) \in \mathbf{R}_{>-\delta_{0}}^{r}$ 
Proof. Denote by $N_{\mathbf{R}}(I)$ the minimal R-subspace of $N_{\mathbf{R}}$ spanned by all $e$ with $\mathbf{R}_{\geq 0} e$ contained in the set of 1-dimensional cones in $\cup_{i \in I(L)} \Sigma_{i}(1)$. Let $\Sigma(L)$ be the complete $G$-invariant fan of cones in $N_{\mathbf{R}}(I)$ which consists of intersections of cones in $\Sigma \subset N_{\mathbf{R}}$ with the subspace $N_{\mathbf{R}}(I)$. Since $\Sigma(L)$ is not necessary a regular fan, we construct a new $G$-invariant fan $\tilde{\Sigma}(L)$ by subdivision of cones in $\Sigma(L)$ into regular ones using the method of Brylinski [4]. This reduces the computation of the local intergral to the one made for local height functions on smooth toric varieties in [2], theorem 2.2.6.

Let $\sigma_{1}, \ldots, \sigma_{\tilde{n}}$ be the set of representatives of $G_{v^{-}}$orbits in the set of 1-dimensional cones in $\tilde{\Sigma}(L) \subset N_{\mathbf{R}}(I)$. We obtain

$$
\begin{gathered}
\int_{T_{I}\left(F_{v}\right)} H_{\Sigma, v}\left(x_{v},(-\mathbf{s},-\mathbf{t})\right) d \alpha_{v}= \\
=Q_{\tilde{\Sigma}(L)}\left(q_{v}^{-l_{\sigma_{1}}(\mathbf{s}, \mathbf{t})}, \ldots, q_{v}^{-l_{\tilde{n}}(\mathbf{s}, \mathbf{t})}\right) \prod_{j=1}^{\tilde{n}}\left(1-\frac{1}{q_{v}^{-l_{\sigma_{j}}(\mathbf{s}, \mathbf{t})}}\right),
\end{gathered}
$$

where $l_{\sigma}(\mathbf{s}, \mathbf{t})$ are linear forms which are $\geq 1-\varepsilon_{0}$ in the domain $\operatorname{Re}(\mathbf{s}, \mathbf{t}) \in \mathbf{R}_{>-\delta_{0}}^{r}$, and $Q_{\tilde{\Sigma}(L)}(\mathbf{z})$ is a polynomial in the variables $\mathbf{z}=$ $\left(z_{1}, \ldots, z_{\tilde{n}}\right)$ such that all monomials in $Q_{\tilde{\Sigma}(L)}(\mathbf{z})-1$ have degree $\geq 2$. Now we notice that $l_{\sigma}(\mathbf{0}, \mathbf{0})=1$ iff $\sigma$ is a 1-dimensional cone in $\Sigma$ and therefore, the cone $\mathbf{R}_{\geq 0} e_{i}$ for some $i \in I(L)$ is contained in the $G_{v^{-}}$orbit of $\sigma$ (see 5.11).

Lemma 5.11. The set of lattice vectors $e \in N$ such that $a(L) \varphi_{L}(e)=$ 1 coincides with the set of lattice vectors $e_{i} \in N_{\mathbf{R}}(I)$ with $\mathbf{R}_{\geq 0} e_{i} \in \Sigma(1)$ and $a(L) \varphi_{L}\left(e_{i}\right)=1$.

Proof. Let $e$ be a lattice point in $N$. Since $\Sigma$ is complete, there exists a $d$-dimensional cone $\sigma \in \Sigma$ such that $e \in \sigma$. We claim that the property $a(L) \varphi_{L}(e)=1$ implies that $e$ is a generator of a 1-dimensional face of $\sigma$. Indeed, we have $a(L) \varphi_{L}(x) \geq \varphi_{\Sigma}(x)$ for all $x \in N_{\mathbf{R}}$. On the other hand, $\sigma$ is generated by a basis of $N$ and $\varphi_{\Sigma}$ has value 1 on these generators. Hence, $e$ must be one of the generators of $\sigma$.

It remains to show that the property $a(L) \varphi_{L}\left(e_{i}\right)=1$ for some generator $e_{i}$ of a 1-dimensional cone $\mathbf{R}_{\geq 0} e_{i} \in \Sigma$ implies that $e_{i} \in N_{\mathbf{R}}(L)$. But this follows from the definition of $N_{\mathbf{R}}(L)$ as the subspace in $N_{\mathbf{R}}$ generated by all elements $e_{i} \in N$ such that $\mathbf{R}_{\geq 0} e_{i} \in \Sigma$ and $a(L) \varphi_{L}\left(e_{i}\right)=1$.

Theorem 5.12. There exists a $\delta>0$ such that the zeta function $Z_{\Sigma}(T, \mathcal{L}, s)$ obtained by restriction of the zeta function $Z_{\Sigma}(T, \mathbf{s})$ to the 
complex line $s[L] \in \operatorname{Pic}\left(X_{\Sigma}\right)_{\mathbf{C}}$ has a representation of the form

$$
Z_{\Sigma}(T, \mathcal{L}, s)=\frac{\Theta_{\mathcal{L}}(\Sigma)}{(s-a(L))^{b(L)}}+\frac{h(s)}{(s-a(L))^{b(L)-1}}
$$

with some function $h(s)$ which is holomorphic in the domain $\operatorname{Re}(s)>$ $a(L)-\delta$ and a nonzero constant $\Theta_{\mathcal{L}}(\Sigma)$.

\section{Appendix: $\mathcal{X}$-FUnCtions of polyhedral CONES}

Let $\left(A, A_{\mathbf{R}}, \Lambda\right)$ be a triple consisting of a free abelian group $A$ of rank $k$, a $k$-dimensional real vector space $A_{\mathbf{R}}=A \otimes \mathbf{R}$ containing $A$ as a sublattice of maximal rank, and a convex $k$-dimensional finitely generated polyhedral cone $\Lambda \subset A_{\mathbf{R}}$ such that $\Lambda \cap-\Lambda=0 \in A_{\mathbf{R}}$. Denote by $\Lambda^{\circ}$ the interior of $\Lambda$ and by $\Lambda_{\mathbf{C}}^{\circ}=\Lambda^{\circ}+i A_{\mathbf{R}}$ the complex tube domain over $\Lambda^{\circ}$. Let $\left(A^{*}, A_{\mathbf{R}}^{*}, \Lambda^{*}\right)$ be the triple consisting of the dual abelian $\operatorname{group} A^{*}=\operatorname{Hom}(A, \mathbf{Z})$, the dual real vector space $A_{\mathbf{R}}^{*}=\operatorname{Hom}\left(A_{\mathbf{R}}, \mathbf{R}\right)$, and the dual cone $\Lambda^{*} \subset A_{\mathbf{R}}^{*}$. We normalize the Haar measure dy on $A_{\mathbf{R}}^{*}$ by the condition: $\operatorname{vol}\left(A_{\mathbf{R}}^{*} / A^{*}\right)=1$.

Definition 6.1. The $\mathcal{X}$-function of $\Lambda$ is defined as the integral

$$
\mathcal{X}_{\Lambda}(\mathbf{s})=\int_{\Lambda^{*}} e^{-\langle\mathbf{s}, \mathbf{y}\rangle} \mathbf{d} \mathbf{y},
$$

where $\mathbf{s} \in \Lambda_{\mathbf{C}}^{\circ}$.

Proposition 6.2. One has $\mathcal{X}_{\Lambda}(\mathbf{s})$ is a rational function

$$
\mathcal{X}_{\Lambda}(\mathrm{s})=\frac{P(\mathbf{s})}{Q(\mathrm{~s})}
$$

where $P$ is a homogeneous polynomial, $Q$ is a product of all linear homogeneous forms defining the codimension 1 faces of $\Lambda$, and $\operatorname{deg} P$ $\operatorname{deg} Q=-k$. In particular, if $\left(A, A_{\mathbf{R}}, \Lambda\right)=\left(\mathbf{Z}^{k}, \mathbf{R}^{k}, \mathbf{R}_{\geq 0}^{k}\right)$, then

$$
\mathcal{X}_{\Lambda}(\mathbf{s})=\frac{1}{s_{1} \cdots s_{k}} \text {. }
$$

Proposition 6.3. [3] Let $\left(A, A_{\mathbf{R}}, \Lambda\right)$ and $\left(\tilde{A}, \tilde{A}_{\mathbf{R}}, \tilde{\Lambda}\right)$ be two triples as above, $k=\operatorname{rk} A$ and $\tilde{k}=\operatorname{rk} \tilde{A}$, and $\psi: A \rightarrow \tilde{A}$ a homomorphism of free abelian groups with a finite cokernel $\operatorname{Coker}(\psi)$ (i.e., the corresponding linear mapping of real vector spaces $\psi: A_{\mathbf{R}} \rightarrow \tilde{A}_{\mathbf{R}}$ is surjective), and $\psi(\Lambda)=\tilde{\Lambda}$. Let $\Gamma=\operatorname{Ker} \psi \subset A$, dy the Haar measure on $\Gamma_{\mathbf{R}}=\Gamma \otimes \mathbf{R}$ normalized by the condition $\operatorname{vol}\left(\Gamma_{\mathbf{R}} / \Gamma\right)=1$. Then for all $\mathbf{s}$ with $\operatorname{Re}(\mathbf{s}) \in \Lambda^{\circ}$ the following formula holds:

$$
\mathcal{X}_{\tilde{\Lambda}}(\psi(\mathbf{s}))=\frac{1}{(2 \pi)^{k-\tilde{k}}|\operatorname{Coker}(\psi)|} \int_{\Gamma_{\mathbf{R}}} \mathcal{X}_{\Lambda}(\mathbf{s}+i \mathbf{y}) \mathbf{d y} \mathbf{y}
$$


where $|\operatorname{Coker}(\psi)|$ is the order of the finite abelian group $\operatorname{Coker}(\psi)$.

Assume that a $\tilde{k}$-dimensional rational finite polyhedral cone $\tilde{\Lambda} \subset \tilde{A}_{\mathbf{R}}$ contains exactly $r$ one-dimensional faces with primitive lattice generators $a_{1}, \ldots, a_{r} \in \tilde{A}$. We set $k:=r, A:=\mathbf{Z}^{r}$ and denote by $\psi$ the natural homomorphism of lattices $\mathbf{Z}^{r} \rightarrow \tilde{A}$ which sends the standard basis of $\mathbf{Z}^{r}$ into $a_{1}, \ldots, a_{r} \in \tilde{A}$, so that $\tilde{\Lambda}$ is the image of the simplicial cone $\mathbf{R}_{\geq 0}^{r} \subset \mathbf{R}^{r}$ under the surjective map of vector spaces $\psi: \mathbf{R}^{r} \rightarrow A_{\mathbf{R}}$. Denote by $\Gamma$ the kernel of $\psi$. By 6.3 we obtain the following:

Corollary 6.4. Let $\mathbf{s}=\left(s_{1}, \ldots, s_{r}\right)$ be the standard coordinates in $\mathbf{C}^{r}$. Then

$$
\mathcal{X}_{\Lambda}(\psi(\mathbf{s}))=\frac{1}{(2 \pi)^{r-k}|\operatorname{Coker}(\psi)|} \int_{\Gamma_{\mathbf{R}}} \frac{1}{\prod_{j=1, n}\left(s_{j}+i y_{j}\right)} \mathbf{d y},
$$

where $\mathbf{d y}$ is the Haar measure on the additive group $\Gamma_{\mathbf{R}}$ normalized by the lattice $\Gamma, y_{j}$ are the coordinates of $\mathbf{y}$ in $\mathbf{R}^{r}$, and $|\operatorname{Coker}(\psi)|$ is the index of the sublattice in $\tilde{A}$ generated by $a_{1}, \ldots, a_{r}$.

\section{REFERENCES}

[1] V. Batyrev, Yu.I.Manin, Sur le nombre des points rationnels de hauteur bornée des variétés algébriques, Math. Ann. 286, (1990), 27-43.

[2] V. Batyrev, Yu. Tschinkel, Rational points of bounded height on compactifications of anisotropic tori, Intern. Math. Research Notices 12, (1995), 591-635.

[3] V. Batyrev, Yu. Tschinkel, Manin's conjecture for toric varieties, Preprint IHES, (1995).

[4] J. L. Brylinski, Décomposition simpliciale d'un réseau, invariante par un group fini d'automorphismes, C.R. Acad. Sci. Paris Sér. A-B 288, (1979), A137-A139.

[5] V.I. Danilov, The geometry of toric varieties. Russ. Math. Surveys 33, (1978), n.2, 97-154.

[6] P.K.J. Draxl, L-Funktionen algebraischer Tori, J. Number Theory 3, (1971), $n^{\circ} 4,444-467$.

[7] M. Köcher, Positivitätsbereiche im $\mathbf{R}^{n}$, Amer. J. Math. 79 (1957), 575-596.

[8] T. Ono, Arithmetic of algebraic tori, Ann. Math. 74, (1961),101-139.

[9] T. Ono, On the Tamagawa number of algebraic tori, Ann. of Math. 78, (1963), 47-73.

[10] E. Peyre, Hauteurs et nombres de Tamagawa sur les variétés de Fano, Duke Math. J. 79 (1995), 101-218.

[11] O.S. Rothaus, Domains of Positivity, Abh. Math. Sem. Univ. Hamburg 24 (1960),189-235.

[12] H. Rademacher, On the Phragmén-Lindelöf principle and some applications, Math. Zeitschrift 72, 192-204, (1959).

[13] E.B. Vinberg, The Theory of Convex Homogeneous Cones, Trans. of Moscow Math. Soc. 12 (1963), 340-403.

[14] V.E. Voskresenskii, Projective invariant Demazure models, Math. USSR Izw. 20(2) (1983), 189-202. 
Universität-GHS-Essen, FAChbereich 6, Mathematik, Universitätsstr. 3, 45141 Essen, Germany

E-mail address: victor.batyrev@uni-essen.de

Dept. of Mathematics, U.I.C., Chichgo, (IL) 60608, U.S.A.

E-mail address: yuri@math.uic.edu 